\title{
Mainstream Smoke Chemistry and In Vitro and In Vivo Toxicity of the Reference Cigarettes 3R4F and 2R4F *
}

by

Ewald Roemer ${ }^{1}$, Heike Schramke ${ }^{1}$, Horst Weiler ${ }^{2}$, Ansgar Buettner ${ }^{3}$, Sandra Kausche ${ }^{2}$, Susanne Weber ${ }^{2}$, An Berges ${ }^{4}$, Markus Stueber ${ }^{2}$, Monja Muench ${ }^{2}$, Edgar Trelles-Sticken ${ }^{2}$, Jan Pype $^{4}$, Karola Kohlgrueber ${ }^{2}$, Hartmut Voelkel ${ }^{2}$, and Sandra Wittke ${ }^{2}$

${ }^{1}$ Philip Morris International Operations, Philip Morris Products S.A., Rue des Usines 90, 2000 Neuchâtel, Switzerland ${ }^{2}$ Philip Morris International R\&D, Philip Morris Research Laboratories GmbH, Fuggerstr. 3, 51149 Cologne, Germany

${ }^{3}$ Histovia GmbH, Schoene Aussicht 5, 51491 Overath, Germany

${ }^{4}$ Philip Morris International R\&D, Philip Morris Research Laboratories bvba, Grauwmeer 14, 3001 Leuven, Belgium

\section{SUMMARY}

A new reference cigarette, the $3 \mathrm{R} 4 \mathrm{~F}$, has been developed to replace the depleting supply of the $2 \mathrm{R} 4 \mathrm{~F}$ cigarette. The present study was designed to compare mainstream smoke chemistry and toxicity of the two reference cigarettes under the International Organization for Standardization (ISO) machine smoking conditions, and to further compare mainstream smoke chemistry and toxicological activity of the $3 \mathrm{R} 4 \mathrm{~F}$ cigarette by two different smoking regimens, i.e., the machine smoking conditions specified by ISO and the Health Canada intensive (HCI) smoking conditions.

The in vitro cytotoxicity and mutagenicity was determined in the neutral red uptake assay, the Salmonella reverse mutation assay, and the mouse lymphoma thymidine kinase assay. Additionally, a 90-day nose-only inhalation study in rats was conducted to assess the in vivo toxicity. The comparison of smoke chemistry between the two reference cigarettes found practically the same yields of total particulate matter (TPM), 'tar', nicotine, carbon monoxide, and most other smoke constituents. For both cigarettes, the in vitro cytotoxicity, mutagenicity, and in vivo toxicity showed the expected smoke-related effects compared to controls without smoke exposure. There were no meaningful differences between the $2 \mathrm{R} 4 \mathrm{~F}$ and $3 \mathrm{R} 4 \mathrm{~F}$ regarding these toxicological endpoints. The assessments for the $3 \mathrm{R} 4 \mathrm{~F}$ cigarette by smoking regimen found as a trivial effect, due to the higher amount of smoke generated per cigarette under HCI conditions, an increased yield of toxicant and higher toxicological activity per cigarette. However, per mg TPM, 'tar', or nicotine, the amounts of toxicants and the in vitro toxicity were generally lower under HCI conditions, but the in vivo activity was not different between the two machine smoking conditions. Overall, as the main result, the present study suggests equivalent smoke chemistry and in vitro and in vivo toxicity for the $2 \mathrm{R} 4 \mathrm{~F}$ and $3 \mathrm{R} 4 \mathrm{~F}$ reference cigarettes. [Beitr. Tabakforsch. Int. 25 (2012) 316-335]

\section{ZUSAMMENFASSUNG}

Eine neue Referenzzigarette 3R4F ist entwickelt worden, die die 2R4F Zigarette ersetzen soll, da deren Vorrat erschöpft ist. In der vorliegenden Studie sollten die Chemie des Hauptstromrauches und die Toxizität beider Referenzzigaretten unter den maschinellen Abrauchbedingungen der Internationalen Organisation für Normung (ISO) verglichen werden. Des Weiteren sollten die Chemie des Hauptstromrauches und dessen Toxizität für die 3R4F Zigarette unter den ISO-Abrauchbedingungen mit denen unter den Bedingungen, wie sie Health Canada (HCI) spezifiziert, verglichen werden.

Die in vitro-Zytotoxizität und -Mutagenität wurden im Neutralrot-Aufnahme-Test, dem Salmonella-Rückmutations-Test und dem Maus-Lymphoma-ThymidinKinase-Test bestimmt. Zusätzlich wurde in einer 90-Tage-Inhalationsstudie an Ratten die in vivo-Toxizität 
bestimmt. Der Vergleich beider Referenzzigaretten ergab keine wesentlichen Unterschiede in der Ausbeute an Partikelphase (TPM), Teer, Nikotin, Kohlenmonoxid und den meisten anderen Rauchbestandteilen. Die in vitro-Zytotoxizität und -Mutagenität und in vivo-Toxizität zeigten, verglichen mit den Kontrollen ohne Rauchexposition, für beide Zigaretten die erwarteten Raucheffekte. Es ergaben sich keine erwähnenswerten Unterschiede zwischen der 2R4F und der 3R4F Zigarette bezüglich dieser toxikologischen Endpunkte. Beim Vergleich der Abrauchbedingungen ergaben sich, bedingt durch die größere Rauchmenge, die unter HCI-Bedingungen generiert wurde, als trivialer Befund auch größere Mengen an toxischen Rauchbestandteilen und eine größere Toxizität pro Zigarette. Hingegen pro mg TPM, Teer oder Nikotin waren die Mengen an toxischen Rauchbestandteilen und die in vitro-Toxizität unter den HCI-Bedingungen im Allgemeinen geringer. Die in vivo-Toxizität war jedoch nicht unterschiedlich unter beiden Abrauchbedingungen.

Insgesamt, als Hauptergebnis, legt diese Untersuchung nahe, dass die Referenzzigaretten $2 \mathrm{R} 4 \mathrm{~F}$ und $3 \mathrm{R} 4 \mathrm{~F}$ bezüglich ihrer Rauchchemie sowie in vitro- und in vivo-Toxizität als gleichartig zu betrachten sind. [Beitr. Tabakforsch. Int. 25 (2012) 316-335]

\section{RESUME}

Une nouvelle cigarette de référence, la $3 \mathrm{R} 4 \mathrm{~F}$, a été développée pour remplacer la 2R4F dont le stock s'épuise. La présente étude vise à comparer le courant principal de la fumée produite par ces deux cigarettes en termes de composition chimique et en termes de toxicité. Dans le cas de la 3R4F la fumée a été produite en suivant le protocole de fumage sur machine préconisé par l'Organisation Internationale de Normalisation (ISO) mais aussi suivant le protocole de fumage sur machine plus intense (HCI) spécifié par Santé-Canada. Ceci a permis de comparer la composition chimique et l'activité toxicologique du courant principal de la fumée de la $3 \mathrm{R} 4 \mathrm{~F}$ obtenu suivant chacun de ces deux régimes de fumage.

La cytotoxicité et la mutagenicité ont été mesurées in-vitro suivant le test de fixation du colorant rouge neutre, le test de mutation réverse de salmonelles et l'épreuve sur cellules de lymphome murin à gène TK. En outre, une étude d'inhalation de 90 jours a été effectuée chez le rat, par voies nasales uniquement, pour évaluer la toxicité in-vivo. Les deux cigarettes de référence donnent des fumées ayant des rendements équivalents en masse totale des particules (TPM), goudron et monoxyde de carbone, ainsi que la majorité des autres analytes. Les mesures de toxicité invitro, cytotoxicité et mutagénicité, ainsi que les tests in-vivo donnent les résultats attendus pour une exposition à la fumée. Aucune différence persuasive n'est observée entre $2 \mathrm{R} 4 \mathrm{~F}$ et $3 \mathrm{R} 4 \mathrm{~F}$ pour ce qui est de ces mesures de toxicité. Comparé à un fumage selon ISO, dans le cas d'un fumage selon le protocole HCI les rendements en produits toxiques ainsi que l'activité toxique de la fumée de la 3R4F sont plus élevés lorsqu'ils sont calculés par cigarette. Ceci est trivial compte tenu de l'accroissement considérable de la quantité de fumée générée. Toutefois, exprimés par unité de masse (mg TPM, mg goudron ou mg nicotine) les rendements en composés toxiques et la toxicité in-vitro sont plus bas que ceux obtenus selon ISO.

Il n'y a pas de différence entre les résultats des mesures de toxicité in-vivo obtenus selon les 2 protocoles de fumage sur machine.

En conclusion, la présente étude suggère principalement une équivalence de la $2 \mathrm{R} 4 \mathrm{~F}$ et de la $3 \mathrm{R} 4 \mathrm{~F}$ en termes de leurs compositions chimiques et de leurs toxicités in-vitro et in-vivo. [Beitr. Tabakforsch. Int. 25 (2012) 316-335]

\section{INTRODUCTION}

Reference cigarettes play an important role in the identification and assessment of cigarette smoke-related effects. These cigarettes allow the replication and comparison of experiments performed in other laboratories. Such comparisons can be performed by setting the values from reference cigarette data to $100 \%$ and expressing the values from other experimental cigarette as a percentage of the reference value. In order to provide reference cigarettes that are easily available for all laboratories, all over the world, working in this field of research, the United States (U.S.) cigarette industry, on request of the Scientific Advisory Board of the Council for Tobacco Research, has provided such cigarettes since 1969 . They resemble typical prototypes of certain market segments. Historically, these cigarettes have also provided the basis for both qualitative and quantitative comparisons of different cigarettes types (1), and they will continue to be necessary in the evaluation of future reduced harm products (2).

The University of Kentucky has provided the organizational structure for the design, development and distribution of reference cigarettes (http://www.ca.uky.edu/refcig/). These reference cigarettes differ in their design and characteristics, e.g., with and without filter, blend composition, and smoke delivery. The cigarettes are constructed to represent typical segments of the American market. One of these reference cigarettes is a "full flavor", filtered, American blended cigarette with a total particulate matter (TPM) yield of approximately $11 \mathrm{mg} /$ cigarette under ISO machine smoking conditions, which is currently in its third version. The first version of this reference, called 1R4F was produced in 1983. It was later replaced in 2003 by its successor the 2R4F cigarette, which was chemically characterized and compared to the $1 \mathrm{R} 4 \mathrm{~F}(3,4)$. Additional smoke chemistry data for the $2 \mathrm{R} 4 \mathrm{~F}$ can be found in studies by ADAM et al. (5) and INTORP et al. (6). The biological activity of its smoke has also been characterized in detail (4, 7-15). In 2008, due to diminishing stock of 2R4F, a replacement was made available. This replacement, the $3 \mathrm{R} 4 \mathrm{~F}$ cigarette has not yet been compared in the literature to the $2 \mathrm{R} 4 \mathrm{~F}$. Evidence that these cigarettes are essentially the same regarding both their smoke chemistry and biological activities would allow for them to be used interchangeably as a comparison basis and would facilitate comparison of a larger number of studies as was the case with the previous versions, $1 \mathrm{R} 4 \mathrm{~F}$ and $2 \mathrm{R} 4 \mathrm{~F}$ (3).

In addition to reference cigarettes, the existence of generally accepted machine smoking protocols is essential to allow for the comparison of results from cigarette smoke obtained in different laboratories. Machine smoking 


\begin{tabular}{|c|c|c|}
\hline \multirow{2}{*}{ Parameter } & \multicolumn{2}{|c|}{ Cigarette } \\
\hline & 2R4F & $3 \mathrm{R} 4 \mathrm{~F}$ \\
\hline \multicolumn{3}{|l|}{ Physical data } \\
\hline Cigarette length (mm) & 84.0 & 84.0 \\
\hline Filter length (mm) & 27.0 & 27.0 \\
\hline Circumference (mm) & 24.9 & 24.5 \\
\hline Cigarette weight $(\mathrm{g})$ & 1.06 & 1.05 \\
\hline Filter ventilation (\%) & 28.0 & 29.0 \\
\hline Paper permeability (sec/50 mL) & 24.0 & 24.0 \\
\hline Resistance to draw $\left(\mathrm{cm} \mathrm{H}_{2} \mathrm{O}\right)$ & 13.4 & 12.8 \\
\hline \multicolumn{3}{|l|}{ Blend composition (\%) } \\
\hline Flue cured & 32.5 & 35.4 \\
\hline Burley & 19.9 & 21.6 \\
\hline Maryland & 1.2 & 1.4 \\
\hline Oriental & 11.1 & 12.1 \\
\hline Reconstituted (Schweitzer process) & 27.1 & 29.6 \\
\hline Sugar (Isosweet ${ }^{\mathrm{TM}}$ ) & 5.3 & 6.4 \\
\hline Glycerol & 2.8 & 2.7 \\
\hline \multicolumn{3}{|l|}{ Filler analysis (\%) } \\
\hline Total alkaloids & 2.3 & 2.1 \\
\hline Reducing sugars & 10.7 & 8.7 \\
\hline Glycerol & 2.4 & 2.4 \\
\hline \multicolumn{3}{|l|}{ Yield data from supplier } \\
\hline Puff count & 9.2 & 9.0 \\
\hline TPM (mg/cig) & 11.7 & 11.0 \\
\hline 'Tar' (mg/cig) & 9.7 & 9.4 \\
\hline Nicotine (mg/cig) & 0.9 & 0.7 \\
\hline Carbon monoxide (mg/cig) & 13.0 & 12.0 \\
\hline
\end{tabular}

protocols such as those defined by the International Organization for Standardization (16) and Health Canada (17) have been implemented by various regulatory authorities to provide consumers and regulators with data on cigarette smoke yields. It should be noted that standardized machine smoking protocols that apply either more- or less-intense smoking parameters can provide only one specific combination of possible settings of characteristics, like certain fixed puff volume, puff duration, and puff frequency, and are not meant to mimic human smoking behavior, nor could they be expected to do so, as each smoker smokes differently and as such, there is no typical human smoker (18-20).

Therefore, the objective of the present study was to compare the $3 \mathrm{R} 4 \mathrm{~F}$ cigarette with its predecessor the $2 \mathrm{R} 4 \mathrm{~F}$ cigarette in smoke chemistry and biological activity under the ISO machine smoking regimen for their interchangeability/similarity, and to further characterize the $3 \mathrm{R} 4 \mathrm{~F}$ between two smoking protocols, the ISO and HCI regimens in smoke chemistry and biological activity.

\section{EXPERIMENTAL}

\section{Cigarettes and mainstream smoke (MS) generation}

The reference cigarettes $3 \mathrm{R} 4 \mathrm{~F}$ and $2 \mathrm{R} 4 \mathrm{~F}$ were obtained from the University of Kentucky, Kentucky Tobacco Research and Development Center. Both are American blended filter cigarettes (for further details see Table 1). They were conditioned following ISO standard 3402 (21), i.e., at least 48 hours at target conditions of $22{ }^{\circ} \mathrm{C} \pm 1{ }^{\circ} \mathrm{C}$ and a relative humidity of $60 \% \pm 3 \%$. MS was generated under ISO machine smoking conditions following ISO Standard 3308 (16), and under HCI smoking conditions (17). Minor deviations were necessary for technical reasons. The cigarettes were smoked on a 20-port Borgwaldt smoking machine (RM20H, Hamburg, Germany) for the in vitro tests, and on 30-port rotary smoking machines (15 ports blocked for HCI protocol) with an active sidestream smoke exhaust (type Philip Morris Research Laboratories (PMRL), SM2000, equipped with a programmable dual-syringe pump (22) for the in vivo studies. In short, puff volume, puff duration, and puff frequency for the ISO smoking conditions were $35 \mathrm{~mL}, 2 \mathrm{~s}$, and $1 / \mathrm{min}$. For the HCI smoking conditions, the respective values were $55 \mathrm{~mL}, 2 \mathrm{~s}$, and $2 / \mathrm{min}$. Under HCI smoking conditions, all cigarette filter ventilation holes were completely covered by tape.

\section{Mainstream smoke chemistry}

MS was generated for both $2 \mathrm{R} 4 \mathrm{~F}$ and $3 \mathrm{R} 4 \mathrm{~F}$ cigarettes under the ISO conditions as described above. In addition, the $3 \mathrm{R} 4 \mathrm{~F}$ was smoked according the HCI specifications. Analytes in smoke were quantified and compared for both cigarettes according to established methodology $(17,23)$ as previously described (9). Total particulate matter (TPM) was determined gravimetrically from the smoke trapped on Cambridge glass fiber filters (23) which were also used for sample collection of individual particle phase analytes (see below). Nicotine was determined by gas chromatography (GC) with flame ionization detection from a 2-propanol extract of the TPM filter. Water was determined from the same 2-propanol extract by Karl Fischer titration (24). Carbon monoxide was determined by non-dispersive infra- 
red photometry (25). 'Tar' yield was calculated as the TPM yield minus the nicotine and water yields (23). Aldehydes, derivatized with 2,4-dinitrophenylhydrazine and stabilized with pyridine, were determined by high-performance liquid chromatography with ultraviolet (HPLC/UV) detection using water/acetonitrile (9:1) and methanol as solvents (26). Vinyl chloride, 1,3-butadiene, isoprene, benzene, toluene, acrylonitrile, and styrene in the gas phase were trapped in three impingers containing methanol at approx. $-78^{\circ} \mathrm{C}$ cooled with 2-propanole and dry ice and analyzed after addition of internal standards by GC using a $\mathrm{CP}$ PoraBond Q column ( $25 \mathrm{~m} \times 0.25 \mathrm{~mm}, 3 \mu \mathrm{m}$ ) coupled to a mass spectrometer (GC-MS) with electron impact ionization in single ion monitoring mode (27). Styrene and acetamide in TPM were extracted from a glass fiber filter using acetone and analyzed after addition of internal standards by GC using a DB-WAX column (30 m x $0.25 \mathrm{~mm}, 0.25 \mu \mathrm{m})$ coupled to a mass spectrometer (GC/MS) with electron impact ionization in single ion monitoring mode. The analysis of acrylamide after extraction from a glass fiber filter was performed as described (28). Ethylene oxide in the gas phase was trapped in an impinger containing toluene at approx. $-78{ }^{\circ} \mathrm{C}$ (cooled with 2-propanole and dry ice) which was connected in series with a glass fiber filter as first trap. After addition of the internal standard propylene oxide- $\mathrm{d}_{6}$, the toluene solution was analyzed by GC using a CP PoraPlot U column ( $25 \mathrm{~m} \times 0.25 \mathrm{~mm}, 8 \mu \mathrm{m})$ and hydrogen as carrier gas coupled to a mass spectrometer (GC-MS) with electron impact ionization in single ion monitoring mode (29). 2-nitro-propane was determined from mainstream smoke trapped on a silica cartridge by adding 2-methyl-2-nitropropane as internal standard, washing the cartridge with pentane and eluting the target analyte using $15 \%$ diethyl ether in n-pentane. 2-nitropropane was analyzed by GC-MS/MS in chemical ionization mode using iso-butane as ionization gas, helium as carrier gas and argon as collision gas. Aromatic amines were determined by extracting TPM-filters with dilute hydrochloric acid, followed by back extraction, derivatization, clean-up by solid phase extraction, and analysis by GC with a triple quadrupole mass spectrometer (30). Nitrogen oxides were determined by online gas phase chemiluminescence according to the CORESTA recommended method (31). Hydrogen cyanide was trapped in two impingers with sodium hydroxide solution connected in series. An aliquot was analyzed by headspace GC with nitrogen sensitive detection after acidification of the samples with phosphoric acid. Ammonia was trapped on a glass fiber filter and a wash bottle connected in series. The glass fiber filter was extracted with the content of the wash bottle, derivatized with dansyl chloride, and analyzed by HPLC with a tandem mass spectrometer (HPLC/MS-MS) (32).

Volatile $N$-nitrosamines were collected on a glass fiber filter and in two wash bottles containing a citrate/phosphate buffer solution with ascorbic acid to inhibit artificial generation of $\mathrm{N}$-nitrosamines. The glass fiber filter was extracted with citrate/phosphate buffer solution with ascorbic acid and combined with the buffer solution of the wash bottles. The combined buffer solution was three times extracted with dichloromethane and the concentrated chloromethane phase was eluted through an alumina column. After elution with dichloromethane and another concentration step, the extract was analyzed by GC with a thermal energy analyzer. Tobacco-specific $N$-nitrosamines (TSNAs) were analyzed as published (33). TSNAs were extracted with ammonium acetate solution from TPM trapped on a glass fiber filter pad, and analyzed by HPLC/MS-MS. Phenols were extracted from a TPM filter with trichloromethane/acetone after addition of the internal standards phenol- $\mathrm{d}_{6}$, catechol- $\mathrm{d}_{6}$ and hydroquinone- $\mathrm{d}_{6}$. An aliquot of the extract was derivatized with $\mathrm{N}, \mathrm{O}$-bis(trimethylsilyl)-trifluoracetamide / $1 \%$ trimethyl-chlorosilane and the trimethylsilyl ethers of the phenols were analyzed by GC-MS using electron impact ionization in single ion monitoring mode. Polycyclic aromatic hydrocarbons were extracted from TPM filters with pentane/isooctane (9:1) after addition of the labeled internal standards. The sample clean-up was performed by a 2 -step solid phase extraction using aminopropyl cartridges eluted with n-hexane, and octadecyl cartridges eluted with methanol. After concentration of the eluate by solvent evaporation and dissolving in isooctane, the 13 target analytes were determined by GC-MS using electron impact ionization in single ion monitoring mode. Arsenic, cadmium, chromium, nickel, lead, and selenium were trapped in quartz glass tubes using electrostatic precipitation. The condensate was dissolved with dichloromethane/methanol mixture, and after addition of nitric acid, hydrogen peroxide, and water, the samples were subjected to microwave digestion and analyzed with atomic absorption spectroscopy. In the case of matrix interferences, selenium was reanalyzed with the flow injection analysis system furnace technique. Mercury, after electrostatic precipitation of the particle phase, was trapped in 2 impingers containing potassium permanganate in sulfuric acid. For microwave digestion hydrogen peroxide was added. The digest was made up with water and an aliquot was analyzed with a mercury analyzer.

\section{In vitro toxicity}

Assessments of cytotoxicity and mutagenicity were carried out for evaluations of 3R4F and 2R4F cigarettes under ISO machine smoking conditions and the $3 \mathrm{R} 4 \mathrm{~F}$ reference cigarette was also assessed according to HCI machine smoking conditions. Cytotoxicity of TPM, and the gas vapor phase $(\mathrm{GVP})$ from the $3 \mathrm{R} 4 \mathrm{~F}$ and $2 \mathrm{R} 4 \mathrm{~F}$ reference cigarettes, was assessed with the neutral red uptake (NRU) assay with mouse embryo BALB/c 3T3 cells as previously described (8). Briefly, $1.6 \times 10^{4}$ cells were seeded and cultivated in culture medium containing $10 \%$ fetal bovine serum (FBS; $100 \mu \mathrm{L}$ per well). Approximately 24 hours after seeding, the cells were exposed for 24 hours to the smoke fractions, suspended or dissolved in culture medium containing 5\% FBS (100 $\mu \mathrm{L}$ per well) resulting in a final concentration of $1.6 \%$ dimethyl sulfoxide (DMSO) and $8.4 \%$ phosphate buffered saline (PBS), or to solvent control (100 $\mu \mathrm{L}$ per well; culture medium containing 5\% FBS, $1.6 \%$ DMSO, and 8.4\% PBS). Following exposure, cells were incubated for 3 hours in culture medium containing $5 \%$ FBS and neutral red dye $(100 \mu \mathrm{L}$ per well). Cells were washed with PBS, and the neutral red dye taken up by viable cells was extracted with a destaining solution 
(ethanol/acetic acid; $100 \mu \mathrm{L}$ per well). The optical density of the neutral red, a measure for the number of viable cells, was determined photometrically at $540 \mathrm{~nm}$. Cytotoxicity assessments were performed in triplicate for each smoke fraction, from both cigarettes, using 8 equidistant smoke fraction concentrations with $2-16 \mathrm{cig} / \mathrm{L}$ (TPM) and 3-24 $\mathrm{cig} / \mathrm{L}$ (GVP) for the reference cigarettes smoked under ISO machine smoking conditions, and 0.7-5.6 (TPM) cig/L and 1-8 cig/L (GVP) for the 3R4F smoked according to the HCI machine smoking conditions.

The Ames Salmonella typhimurium reverse mutation assay was performed in general accordance to the Organization for Economic Co-operation and Development, OECD, guideline no. 471 (1997) using the five tester strains TA98, TA100, TA102, TA1535, and TA1537, with and without the S9 fraction, as previously reported (34). Three TPM concentrations per strain were used, ranging from 0.4-2.5 mg TPM/plate depending on the strain. The S9 fraction was purchased from Cytotest Cell Research (CCR, Rossdorf, Germany), and was prepared from the livers of male Sprague-Dawley rats injected with Aroclor 1254. Bacterial mutagenicity was determined for two independent TPM batches of both reference cigarettes. The number of revertants with and without the metabolic activation system was determined for each mutagenicity assay with an automatic colony counter.

The mouse lymphoma assay (MLA) for the mutagenicity of TPM was performed using L5178Y/tk+/--3.7.2C mouse lymphoma cells essentially according to OECD guideline no. 476 (1997) in the microtiter plate version (35) as previously described (10). Cells were obtained from LGC Standards, Germany (in partnership with American Tissue Culture Collection, Manassas, VA, USA). The assays were performed with two independent TPM batches, at three TPM doses with S9 metabolic activation (80, 140/150, $200 / 220 \mu \mathrm{g} / \mathrm{mL}$ TPM) and without S9 metabolic activation $(25,40,55 \mu \mathrm{g} / \mathrm{mL}$ TPM). S9 metabolic activation mix was obtained from CCR, Rossdorf, Germany.

\section{Inhalation / in vivo toxicity}

Two 90-day nose-only inhalation studies with male and female Sprague-Dawley rats were performed to determine the biological activity of diluted MS. The first study was conducted using an exposure regimen of $6 \mathrm{~h} / \mathrm{day}$, 7 days/week at $200 \mu \mathrm{g} / \mathrm{L}$ TPM of the $2 \mathrm{R} 4 \mathrm{~F}$ or $3 \mathrm{R} 4 \mathrm{~F}$ cigarette. The second study was conducted at an exposure regimen of $6 \mathrm{~h} / \mathrm{d}, 5$ days/week at three increasing concentrations of 100,150 , and $200 \mu \mathrm{g} / \mathrm{L}$ TPM of the $3 \mathrm{R} 4 \mathrm{~F}$ cigarette and compared between the ISO and HCI machine smoking protocols.

Generally, 10 rats/sex/group were exposed nose-only to MS or to filtered, conditioned fresh air (sham-exposure group). General conditions and animal health, as well as smoke exposure and uptake were monitored. Local effects in the respiratory tract along with systemic effects were investigated after 13 weeks of smoke exposure as previously described (36). Endpoints included all parameters specified in the OECD Guideline for Testing of Chemicals 413 (37) with an extended histopathological assessment of irritation in the respiratory tract. Histopathological changes were scored according to a defined severity scale from 0 to
5. Mean severity scores were calculated based on all rats in a group. Hereby, special histological sections were prepared for the nose according to the method of YOUNG (38), and for the larynx according to LEWIS (39). The trachea was cut frontally (at the bifurcation). One frontal section passing through the main bronchus for the left lung and one frontal section passing through a maximum number of lobes for the right lung were prepared (40). A 42-day post-inhalation period was included for the high-dose smoke exposure and the sham-exposure groups, to assess reversibility, persistence, or delayed occurrence of smokeexposure effects (data not shown).

\section{Statistical analysis}

All tests were conducted without correction for multiple comparisons. The significance level is $\alpha \leq 0.05$, with the exception of smoke chemistry tests which were conducted at the significance levels of $0.05,0.01$, and 0.001 . Generally, the mean and the standard error were given as descriptive statistics. Comparisons were made on a per cigarette, a per milligram TPM, a per milligram 'tar', and a per milligram nicotine basis.

For smoke chemistry, comparisons were performed using the t-test. For the NRU assay, the reciprocal $\mathrm{EC}_{50}\left(1 / \mathrm{EC}_{50}\right)$ was determined separately for each smoke fraction (TPM and GVP) and for each of the three batches. The mean reciprocal $\mathrm{EC}_{50}$ values were compared by t-test. The Ames assay data were evaluated based on the slopes of the linear dose-response curves using linear regression analysis with Poisson-weighted data excluding the 0 -dose as previously described (8). Analysis of covariance (ANCOVA) was used to compare slopes. MLA assay data was compared using the dose-response curves for the mutant frequency values as calculated by nonlinear regression analysis with the power function $\mathrm{y}=\mathrm{a}+\mathrm{bx}^{\mathrm{c}}$ from which the smoke concentrations were calculated that resulted in a mutagenic response as high as three times the background (spontaneous) mutant frequency $\left(\mathrm{C}_{3 \mathrm{~B}}\right)$. In addition, nonlinear dose-response curves were examined using the sum of square reduction test (comparison 3R4F ISO/HCI only). In the 90-day rat inhalation studies, MS exposure groups were compared with the sham-exposure group using one-way ANOVA, followed by the Dunnett post-hoc test to confirm exposure effects. Differences in biological activity due to the different smoking protocols for all endpoints were compared on a per milligram TPM basis using twoway ANOVA. Histopathological findings from nonrespiratory organs were evaluated with a scoring system from 0 to 5 and analyzed either with the Cochran Mantel Haenszel test for overall and pair-wise comparisons or by ANOVA. Incidences were analyzed with $\chi^{2}$ statistics.

\section{RESULTS}

\section{Cigarette smoke chemistry}

In the comparisons of the $3 \mathrm{R} 4 \mathrm{~F}$ with the $2 \mathrm{R} 4 \mathrm{~F}$ reference cigarette analyte, yields smoked according to ISO machine smoking conditions, the mean difference over all calculation bases was less than $3 \%$ between both cigarettes 
Table 2. Relative smoke constituent yields of the $3 R 4 F$ versus the $2 R 4 F$ reference cigarette, when smoked according to ISO machine smoking conditions.

\begin{tabular}{|c|c|c|c|c|}
\hline \multirow{2}{*}{ Parameter } & \multicolumn{4}{|c|}{$3 \mathrm{R} 4 \mathrm{~F}$ to $2 \mathrm{R} 4 \mathrm{~F}$ ratio $(\%)$} \\
\hline & Per cigarette & Per mg TPM & Per mg 'tar' & Per mg nicotine \\
\hline \multicolumn{5}{|l|}{ ISO Parameter } \\
\hline TPM & $105^{\star \star}$ & 100 & 99 & 101 \\
\hline ‘Tar’ & $105^{\star \star *}$ & 101 & 100 & 101 \\
\hline Nicotine & $104^{*}$ & 99 & 99 & 100 \\
\hline Water & 100 & 95 & 95 & 96 \\
\hline $\mathrm{CO}$ & $105^{\star *}$ & 100 & 99 & 100 \\
\hline \multicolumn{5}{|l|}{ Aldehydes } \\
\hline Formaldehyde & 109 & 104 & 103 & 104 \\
\hline Acetaldehyde & 105 & 100 & 99 & 100 \\
\hline Acrolein & 104 & 99 & 98 & 99 \\
\hline Propionaldehyde & 103 & 98 & 98 & 99 \\
\hline Crotonaldehyde & $112^{*}$ & 107 & 106 & 108 \\
\hline \multicolumn{5}{|l|}{ Aliphatic dienes } \\
\hline 1,3-Butadiene & 99 & 94 & 94 & 95 \\
\hline Isoprene & 96 & $92^{*}$ & $91^{*}$ & $92^{*}$ \\
\hline \multicolumn{5}{|l|}{ Acid derivatives } \\
\hline Acetamide & 102 & 97 & 97 & 98 \\
\hline Acrylamide & $87^{\star *}$ & $83^{\star * *}$ & $83^{\star * *}$ & $84^{\star * *}$ \\
\hline Acrylonitrile & 100 & 95 & 95 & 96 \\
\hline \multicolumn{5}{|l|}{ Epoxides } \\
\hline Ethylene oxide & 97 & 92 & 92 & 93 \\
\hline \multicolumn{5}{|l|}{ Nitro compounds } \\
\hline 2-Nitropropane & 90 & 86 & 85 & 86 \\
\hline \multicolumn{5}{|l|}{ Aromatic amines } \\
\hline o-Toluidine & 101 & 96 & 96 & 97 \\
\hline o-Anisidine & 104 & 99 & 99 & 100 \\
\hline 2-Naphthylamine & 98 & $93^{*}$ & $93^{*}$ & 94 \\
\hline 4-Aminobiphenyl & 97 & $93^{*}$ & $92^{*}$ & 93 \\
\hline \multicolumn{5}{|l|}{ Halogen compounds } \\
\hline Vinyl chloride & $87^{*}$ & $83^{*}$ & $82^{*}$ & $83^{*}$ \\
\hline \multicolumn{5}{|c|}{ Inorganic compounds } \\
\hline Nitrogen oxides & 98 & $94^{*}$ & $93^{*}$ & $94^{*}$ \\
\hline Hydrogen cyanide & 102 & 97 & 96 & 97 \\
\hline Ammonia & 90 & 85 & 85 & 86 \\
\hline \multicolumn{5}{|c|}{ Monocyclic aromatic hydrocarbons } \\
\hline Benzene & 98 & $94^{*}$ & $93^{*}$ & 94 \\
\hline Toluene & 100 & 95 & 95 & 96 \\
\hline Styrene & 99 & 94 & 93 & 94 \\
\hline \multicolumn{5}{|c|}{ Volatile $N$-nitrosamines } \\
\hline NDMA & $<\mathrm{LOQ}$ & $<L O Q$ & $<L O Q$ & $<\mathrm{LOQ}$ \\
\hline NMEA & $<\mathrm{LOQ}$ & $<\mathrm{LOQ}$ & $<\mathrm{LOQ}$ & $<L O Q$ \\
\hline NDEA & $<\mathrm{LOQ}$ & $<L O Q$ & $<L O Q$ & $<L O Q$ \\
\hline NPRA & $<\mathrm{LOQ}$ & $<L O Q$ & $<L O Q$ & $<\mathrm{LOQ}$ \\
\hline NBUA & $<L O Q$ & $<L O Q$ & $<L O Q$ & $<\mathrm{LOQ}$ \\
\hline NPY & $<L O Q$ & $<L O Q$ & $<L O Q$ & $<\mathrm{LOQ}$ \\
\hline NPI & $<L O Q$ & $<\mathrm{LOQ}$ & $<L O Q$ & $<L O Q$ \\
\hline \multicolumn{5}{|c|}{ Tobacco-specific N-nitrosamines } \\
\hline NNN & $84^{\star * *}$ & $80^{\star * *}$ & $79^{* * *}$ & $80^{* * *}$ \\
\hline NNK & $78^{\star * *}$ & $74^{* * *}$ & $74^{* * *}$ & $75^{\star * *}$ \\
\hline NAB & 103 & 98 & 98 & 99 \\
\hline NAT & 106 & 101 & 100 & 101 \\
\hline
\end{tabular}


Table 2. (cont.).

\begin{tabular}{|c|c|c|c|c|}
\hline \multirow{2}{*}{ Parameter } & \multicolumn{4}{|c|}{ 3R4F to $2 \mathrm{R} 4 \mathrm{~F}$ ratio $(\%)$} \\
\hline & Per cigarette & Per mg TPM & Per mg 'tar' & Per mg nicotine \\
\hline \multicolumn{5}{|l|}{ Phenols } \\
\hline Phenol & $108^{*}$ & 103 & 102 & 104 \\
\hline Catechol & 102 & 98 & 97 & 98 \\
\hline Hydroquinone & $106^{\star * *}$ & 101 & 101 & 102 \\
\hline \multicolumn{5}{|c|}{ Polycyclic aromatic hydrocarbons } \\
\hline Pyrene & $108^{*}$ & 103 & 102 & 103 \\
\hline Benz[a]anthracene & 105 & 101 & 100 & 101 \\
\hline Benzo[b]fluoranthene & $113^{\star * *}$ & $107^{*}$ & $107^{*}$ & $108^{*}$ \\
\hline Benzo[]]fluoranthene & $113^{* *}$ & $108^{*}$ & $107^{*}$ & $109^{*}$ \\
\hline Benzo[k]fluoranthene & $116^{* *}$ & $111^{*}$ & $110^{*}$ & $111^{*}$ \\
\hline Benzo[a]pyrene & $108^{* *}$ & 103 & 103 & $104^{*}$ \\
\hline Dibenz $[a, h]$ anthracene & $<L O Q$ & $<L O Q$ & $<\mathrm{LOQ}$ & $<L O Q$ \\
\hline Dibenzo[a,e]pyrene & 108 & 103 & 103 & 104 \\
\hline Dibenzo[a, $h$ ]pyrene & $<L O Q$ & $<L O Q$ & $<L O Q$ & $<L O Q$ \\
\hline Dibenzo[a,i]pyrene & $<L O Q$ & $<L O Q$ & $<L O Q$ & $<\mathrm{LOQ}$ \\
\hline Dibenzo[a,/]pyrene & $<L O Q$ & $<L O Q$ & $<L O Q$ & $<L O Q$ \\
\hline Indeno[1,2,3-cd]pyrene & $113^{* * *}$ & $107^{\star \star}$ & $107^{\star *}$ & $108^{\star *}$ \\
\hline 5-Methylchrysene & $<L O Q$ & $<L O Q$ & $<\mathrm{LOQ}$ & $<L O Q$ \\
\hline \multicolumn{5}{|l|}{ Elements } \\
\hline Cadmium & 99 & 95 & 94 & 95 \\
\hline Chromium & 105 & 100 & 100 & 101 \\
\hline Nickel & $<\mathrm{LOQ}$ & $<L O Q$ & $<L O Q$ & $<\mathrm{LOQ}$ \\
\hline Lead & 93 & $89^{\star \star \star}$ & $89^{\star \star \star}$ & $89^{\star *}$ \\
\hline Arsenic & $109^{\star \star *}$ & $104^{*}$ & $103^{*}$ & $104^{*}$ \\
\hline Selenium & 102 & 98 & 97 & 98 \\
\hline Mercury & $90^{* *}$ & 86 & 85 & 86 \\
\hline
\end{tabular}

$\mathrm{CO}=$ carbon monoxide; $\mathrm{LOQ}=$ at least one value below limit of quantification; NBUA = N-nitrosodi-n-butylamine; NDMA = $N$ nitrosodimethylamine; NDEA = N-nitroso-n-diethylamine; NMEA = N-methylethanolamine; NNN = N'-nitrosonornicotine; NNK = 4(methylnitrosamino)-1-(3-pyridyl)-1-butanone; NPI = N-nitrosopiperidine; NPRA = N-nitrosodi-n-propylamine; NPY = N-nitrosopyrrolidine; TPM $=$ total particulate matter.

Statistical significances: ${ }^{*}: 0.01<p \leq 0.05,{ }^{* *}: 0.001<p \leq 0.01,{ }^{* *}: p \leq 0.001$.

(Table 2; Figure 1; Annex, Table A). The maximum differences were $+16 \%$ (benzo $[k]$ fluoroanthene, per cigarette) and $-26 \%$ (NNK, per mg TPM and 'tar'). Statistically significant lower yields for the $3 \mathrm{R} 4 \mathrm{~F}$ cigarettes were found for acrylamide, ammonia, vinyl chloride, and the TSNA $\mathrm{N}$-nitrosonornicotine (NNN) and 4-(methyl-nitro-samino)1-(3-pyridyl)-1-butanone (NNK) using all calculation bases (per cigarette, per mg 'tar', TPM or nicotine). Statistically significant higher yields for the 3R4F reference cigarette were found for 4 PAHs and arsenic for all calculation bases. Other statistically significant differences were well within the inherent variability of the analytes yields and not observable under all calculation bases.

Further comparisons of smoke chemistry for the 3R4F reference cigarette were performed under the ISO and the HCI machine smoking conditions. Yield ratios for each calculation basis are presented in Table 3. As a trivial finding, due to the higher amount of smoke produced under the HCI machine smoking conditions, statistically significant higher yields per cigarette were found for all constituents under the HCI compared to the ISO machine smoking conditions $(\mathrm{p} \leq 0.001)$. However, when expressed per mg TPM, per mg 'tar', and per mg nicotine the yields of TSNAs, phenols, nitrogen oxide, aromatic amines, and benzene, were generally lower under HCI machine smoking conditions, as compared to when using the ISO regimen ( $\mathrm{p} \leq 0.05$; for all calculation bases). Polycyclic aromatic hydrocarbon levels were lower under the HCI compared to the ISO machine smoking conditions ( $\mathrm{p} \leq$ 0.01 ) on a per mg TPM basis and on a per mg 'tar' basis

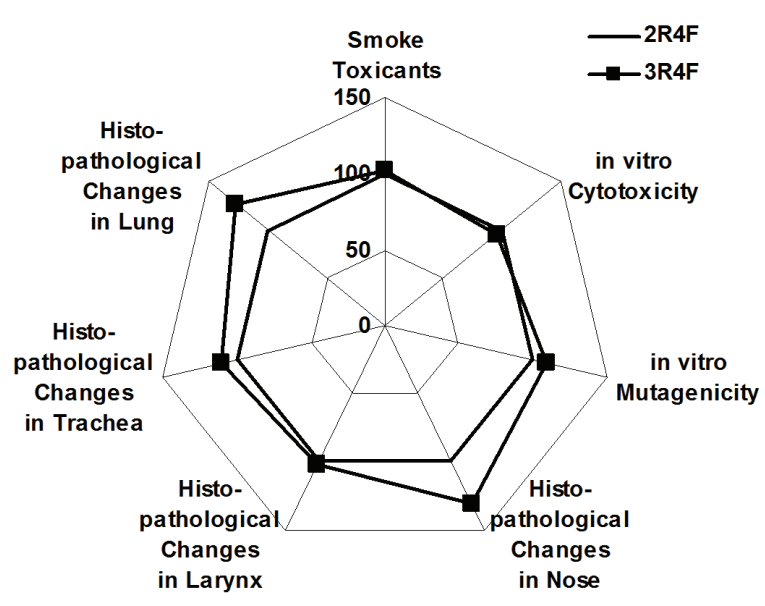

Figure 1. Summary of comparisons of the $3 R 4 F$ to $2 R 4 F$ reference cigarettes - means from Tables 2,4 , and 6 . 
Table 3. Relative smoke constituent yields of the 3R4F reference cigarette when smoked according to ISO and $\mathrm{HCI}$ machine smoking conditions.

\begin{tabular}{|c|c|c|c|c|}
\hline \multirow{2}{*}{ Parameter } & \multicolumn{4}{|c|}{$3 \mathrm{R} 4 \mathrm{~F} \mathrm{HCl}$ to $3 \mathrm{R} 4 \mathrm{~F}$ ISO ratio (\%) } \\
\hline & Per cigarette & Per mg TPM & Per mg 'tar' & Per mg nicotine \\
\hline \multicolumn{5}{|l|}{ ISO Parameter } \\
\hline TPM & $367^{* * * a}$ & 100 & $119^{\star * *}$ & $136^{* * *}$ \\
\hline ‘Tar' & $308^{\star \star \star}$ & $84^{* \star \star}$ & 100 & $114^{* * *}$ \\
\hline Nicotine & $271^{* * *}$ & $74^{\star * *}$ & $88^{* * *}$ & 100 \\
\hline Water & $796^{\star \star *}$ & $217^{* \star *}$ & $258^{\star * *}$ & $294^{* \star *}$ \\
\hline $\mathrm{CO}$ & $279^{* \star *}$ & $76^{\star \star *}$ & $91^{* * *}$ & 103 \\
\hline \multicolumn{5}{|l|}{ Aldehydes } \\
\hline Formaldehyde & $338^{\star \star *}$ & $92^{\star \star \star}$ & 110 & $125^{\star \star *}$ \\
\hline Acetaldehyde & $274^{* \star * a}$ & $75^{\star \star *}$ & $89^{* *}$ & 101 \\
\hline Acrolein & $298^{\star * \star a}$ & $81^{* * *}$ & 97 & $110^{* \star *}$ \\
\hline Propionaldehyde & $267^{\star * \star a}$ & $73^{* * *}$ & $87^{* \star *}$ & 98 \\
\hline Crotonaldehyde & $415^{\star \star \star}$ & $113^{*}$ & $135^{\star * *}$ & $153^{* * *}$ \\
\hline \multicolumn{5}{|l|}{ Aliphatic dienes } \\
\hline 1,3-Butadiene & $272^{* * *}$ & $74^{* *}$ & 88 & 100 \\
\hline Isoprene & $280^{* * *}$ & $76^{* *}$ & 91 & 103 \\
\hline \multicolumn{5}{|l|}{ Acid derivatives } \\
\hline Acetamide & $407^{* * *}$ & $111^{*}$ & $132^{* * *}$ & $150^{* * *}$ \\
\hline Acrylonitrile & $273^{* * *}$ & $74^{\star * *}$ & $89^{*}$ & 101 \\
\hline \multicolumn{5}{|l|}{ Nitro compounds } \\
\hline 2-Nitropropane & $244^{* * *}$ & $67^{* \star *}$ & $79^{* *}$ & 90 \\
\hline \multicolumn{5}{|l|}{ Aromatic amines } \\
\hline o-Toluidine & $223^{* * *}$ & $61^{* * *}$ & $72^{\star \star *}$ & $82^{* * *}$ \\
\hline o-Anisidine & $231^{\star \star * a}$ & $63^{* \star *}$ & $75^{\star \star \star}$ & $85^{\star \star}$ \\
\hline 2-Naphthylamine & $203^{* * *}$ & $55^{\star \star *}$ & $66^{\star \star *}$ & $75^{\star \star *}$ \\
\hline 4-Aminobiphenyl & $248^{\star * *}$ & $68^{* * *}$ & $81^{* \star}$ & 92 \\
\hline \multicolumn{5}{|l|}{ Halogen compounds } \\
\hline Vinyl chloride & $249^{* * *}$ & $68^{* \star *}$ & $81^{* *}$ & 92 \\
\hline \multicolumn{5}{|l|}{ Inorganic compounds } \\
\hline Nitrogen oxides & $246^{\star \star *}$ & $67^{* \star *}$ & $80^{\star * *}$ & $91^{* *}$ \\
\hline Hydrogen cyanide & $427^{* \star \star}$ & $116^{*}$ & $139^{* * *}$ & $158^{* \star *}$ \\
\hline \multicolumn{5}{|c|}{ Monocyclic aromatic hydrocarbons } \\
\hline Benzene & $241^{* * *}$ & $66^{\star \star \star}$ & $78^{\star \star \star}$ & $89^{*}$ \\
\hline Toluene & $257^{\star \star \star *}$ & $70^{* \star \star}$ & $83^{* *}$ & 95 \\
\hline Styrene & $418^{* * * a}$ & $114^{*}$ & $136^{* *}$ & $154^{* \star *}$ \\
\hline \multicolumn{5}{|c|}{ Volatile N-nitrosamines } \\
\hline NDMA & $<\mathrm{LOQ}$ & $<\mathrm{LOQ}$ & $<\mathrm{LOQ}$ & $<\mathrm{LOQ}$ \\
\hline NMEA & $<L O Q$ & $<\mathrm{LOQ}$ & $<\mathrm{LOQ}$ & $<\mathrm{LOQ}$ \\
\hline NDEA & $<\mathrm{LOQ}$ & $<\mathrm{LOQ}$ & $<\mathrm{LOQ}$ & $<\mathrm{LOQ}$ \\
\hline NPRA & $<\mathrm{LOQ}$ & $<\mathrm{LOQ}$ & $<\mathrm{LOQ}$ & $<\mathrm{LOQ}$ \\
\hline NBUA & $<\mathrm{LOQ}$ & $<\mathrm{LOQ}$ & $<\mathrm{LOQ}$ & $<\mathrm{LOQ}$ \\
\hline NPY & $<\mathrm{LOQ}$ & $<\mathrm{LOQ}$ & $<\mathrm{LOQ}$ & $<\mathrm{LOQ}$ \\
\hline NPI & $<\mathrm{LOQ}$ & $<\mathrm{LOQ}$ & $<\mathrm{LOQ}$ & $<\mathrm{LOQ}$ \\
\hline \multicolumn{5}{|c|}{ Tobacco-specific N-nitrosamines } \\
\hline NNN & $249^{* * *}$ & $68^{* * *}$ & $81^{* * *}$ & $92^{* *}$ \\
\hline NNK & $268^{* * *}$ & $73^{* * *}$ & $87^{\star \star}$ & 99 \\
\hline NAB & $231^{* * *}$ & $63^{* * *}$ & $75^{\star * *}$ & $85^{\star *}$ \\
\hline NAT & $250^{* \star *}$ & $68^{* \star *}$ & $81^{* * *}$ & $92^{*}$ \\
\hline \multicolumn{5}{|l|}{ Phenols } \\
\hline Phenol & $203^{* \star *}$ & $55^{\star \star \star}$ & $66^{\star * *}$ & $75^{\star \star \star}$ \\
\hline Catenol & $251^{* * *}$ & $68^{* * *}$ & $81^{* * *}$ & $93^{* *}$ \\
\hline Hydroquinone & $269^{\star \star \star ~ a ~}$ & $73^{* \star *}$ & $87^{* * *}$ & 99 \\
\hline o-Cresol & $196^{\star * \star}$ & $53^{* \star *}$ & $64^{\star \star *}$ & $72^{\star \star \star}$ \\
\hline$m$-Cresol & $196^{\star \star *}$ & $53^{* \star *}$ & $64^{* * *}$ & $72^{\star \star \star}$ \\
\hline
\end{tabular}


Table 3. (cont.).

\begin{tabular}{|c|c|c|c|c|}
\hline \multirow{2}{*}{ Parameter } & \multicolumn{4}{|c|}{$3 \mathrm{R} 4 \mathrm{~F} \mathrm{HCl}$ to $3 \mathrm{R} 4 \mathrm{~F}$ ISO ratio (\%) } \\
\hline & Per cigarette & Per mg TPM & Per mg 'tar' & Per mg nicotine \\
\hline \multicolumn{5}{|l|}{ Phenols } \\
\hline p-Cresol & $207^{\star \star *}$ & $56^{* * *}$ & $67^{* * *}$ & $76^{\star * *}$ \\
\hline Resorcinol & $286^{\star * *}$ & $78^{* * *}$ & $93^{*}$ & 106 \\
\hline \multicolumn{5}{|c|}{ Polycyclic aromatic hydrocarbons } \\
\hline Pyrene & $248^{* * *}$ & $67^{* * *}$ & $80^{* *}$ & 91 \\
\hline Benz[a]anthracene & $260^{* * *}$ & $71^{* * *}$ & $84^{* * *}$ & 96 \\
\hline Benzo $[b]$ fluoranthene & $263^{\star \star *}$ & $72^{* * \star}$ & $85^{\star \star}$ & 97 \\
\hline Benzo[j]fluoranthene & $264^{\star * \star a}$ & $72^{* * *}$ & $86^{\star *}$ & 97 \\
\hline Benzo $[k]$ fluoranthene & $262^{* \star *}$ & $71^{* * *}$ & $85^{\star *}$ & 97 \\
\hline Benzo[a]pyrene & $250^{* \star * a}$ & $68^{* * *}$ & $81^{* * *}$ & $92^{*}$ \\
\hline Dibenz $[a, h]$ anthracene & $<\mathrm{LOQ}$ & $<\mathrm{LOQ}$ & $<\mathrm{LOQ}$ & $<\mathrm{LOQ}$ \\
\hline Dibenzo[a,e]pyrene & $253^{* * *}$ & $69^{* * *}$ & $82^{* * *}$ & $93^{*}$ \\
\hline Dibenzo[a, $h]$ pyrene & $<\mathrm{LOQ}$ & $<L O Q$ & $<\mathrm{LOQ}$ & $<\mathrm{LOQ}$ \\
\hline Dibenzo[a,i]pyrene & $<\mathrm{LOQ}$ & $<L O Q$ & $<\mathrm{LOQ}$ & $<\mathrm{LOQ}$ \\
\hline Dibenzo[a,l]pyrene & $<\mathrm{LOQ}$ & $<\mathrm{LOQ}$ & $<\mathrm{LOQ}$ & $<\mathrm{LOQ}$ \\
\hline Indeno[1,2,3-cd]pyrene & $250^{* \star *}$ & $68^{* \star *}$ & $81^{* * *}$ & $92^{* \star}$ \\
\hline 5-Methylchrysene & $<\mathrm{LOQ}$ & $<\mathrm{LOQ}$ & $<\mathrm{LOQ}$ & $<\mathrm{LOQ}$ \\
\hline \multicolumn{5}{|l|}{ Elements } \\
\hline Arsenic & $378^{* * *}$ & 103 & $123^{* *}$ & $139^{* * *}$ \\
\hline Cadmium & $<\mathrm{LOQ}$ & $<\mathrm{LOQ}$ & $<\mathrm{LOQ}$ & $<\mathrm{LOQ}$ \\
\hline Chromium & $<\mathrm{LOQ}$ & $<L O Q$ & $<\mathrm{LOQ}$ & $<\mathrm{LOQ}$ \\
\hline Nickel & $332^{* * *}$ & 90 & 108 & $122^{*}$ \\
\hline Lead & $314^{\star * *}$ & $85^{*}$ & 102 & 116 \\
\hline
\end{tabular}

$\mathrm{CO}=$ carbon monoxide; $\mathrm{LOQ}=$ at least one value below limit of quantification; NAB $=N$-nitrosoanabasine; NAT = N-nitrosoanatabine; NBUA $=N$-nitrosodi-n-butylamine; NDMA $=N$-nitrosodimethylamine; NDEA $=N$-nitroso-n-diethylamine; NMEA $=N$-methylethanolamine; $N N N=$ $N$-nitrosonornicotine; NNK = 4-(methylnitrosamino)-1-(3-pyridyl)-1-butanone; NPI = N-nitrosopiperidine; NPRA = N-nitrosodi-n-propylamine; $\mathrm{NPY}=N$-nitrosopyrrolidine; TPM $=$ total particulate matter.

a: assumption of equality of variances not reasonable, Satterthwaites approximation of the t-test used,

Statistical significances: ${ }^{*}: 0.01<p \leq 0.05,{ }^{* *}: 0.001<p \leq 0.01,{ }^{* * *}: p \leq 0.001$.

but similar on a per mg nicotine basis. Yields of benzo$[a]$ pyrene, dibenzo $[a, e]$ pyrene, and indeno- $[1,2,3-c, d]-$ pyrene were only significantly lower on a per mg nicotine basis $(\mathrm{p} \leq 0.05)$.

Water, crotonaldehyde, acetamide, hydrogen cyanide and styrene levels were higher for the 3R4F reference cigarette smoked under HCI machine smoking conditions compared to when smoked under ISO conditions ( $\mathrm{p} \leq 0.05)$ regardless of the calculation basis.

\section{In vitro cytotoxicity (NRU assay)}

For all samples there was a reproducible dose-dependent decrease in cell viability for both TPM and GVP exposure of both reference cigarettes. There were no statistically significant differences in the cytotoxicity of either the TPM or GVP smoke fractions of the 3R4F cigarette when compared to that of the $2 \mathrm{R} 4 \mathrm{~F}$ cigarette smoked under the same ISO machine smoking conditions, regardless of the calculation basis (i.e. per cigarette, per unit mass of TPM, 'tar', or nicotine). The mean difference was $-5 \%$ and the maximum difference was $-9 \%$ for GVP on a per cigarette basis (Table 4).

There were significant differences, however, in the cytotoxicity values of the TPM and GVP smoke fractions from the 3R4F reference cigarette when smoked under HCI machine smoking conditions, as compared to the ISO conditions. The expected statistically significant increase in cytotoxicity per cigarette was $+183 \%$ for the TPM and $+198 \%$ for the GVP. A statistically significant decrease in cytotoxicity of $-18 \%$ was observed for the TPM on a per mg TPM basis. The cytotoxicity of the GVP on the same calculation basis was reduced to the same extent, but the difference was not statistically significant. The other calculation bases did not reveal statistical significances (Table 5).

\section{In vitro bacterial mutagenicity (Ames assay)}

Dose-dependent increases in the number of revertants was observed in strains TA98, TA100, and TA1537, with and without S9 metabolic activation, following TPM-exposure from either reference cigarette, compared to solvent control. For these tester strains that, according to the literature (9), have been proven to be responsive to TPM and discriminative, the dose-dependent increases were in most cases statistically significant. There were no statistically significant differences, however, in the observed mutagenicity between the $2 \mathrm{R} 4 \mathrm{~F}$ and $3 \mathrm{R} 4 \mathrm{~F}$ reference cigarettes, when smoked under ISO machine smoking conditions, with and without S9 activation, regardless of the calculation basis (Table 4). On a per cigarette basis, a significantly higher mutagenicity of TPM from the $3 \mathrm{R} 4 \mathrm{~F}$ cigarette $(+98 \%$ to $+263 \%)$ when smoked 
Table 4. Relative in vitro toxicity of the $3 \mathrm{R} 4 \mathrm{~F}$ versus the $2 \mathrm{R} 4 \mathrm{~F}$ reference cigarette, when smoked according to ISO machine smoking conditions.

\begin{tabular}{|c|c|c|c|c|}
\hline \multirow{2}{*}{ Assay, measure, smoke fraction } & \multicolumn{4}{|c|}{$3 \mathrm{R} 4 \mathrm{~F}$ to $2 \mathrm{R} 4 \mathrm{~F}$ ratio (\%) } \\
\hline & Per cigarette & Per mg TPM & Per mg 'tar' & Per mg nicotine \\
\hline \multicolumn{5}{|l|}{ Cytotoxicity, $1 / E C_{50}$} \\
\hline TPM & 96 & 97 & 98 & 99 \\
\hline GVP & 91 & 93 & 93 & 94 \\
\hline \multicolumn{5}{|l|}{ Bacterial mutagenicity, TPM, } \\
\hline TA $98, \quad+S 9$ & 98 & 94 & 94 & 99 \\
\hline TA 100 & 109 & 104 & 104 & 106 \\
\hline TA1537, +S9 & 122 & 117 & 117 & 119 \\
\hline TA 100, - S9 & 125 & 121 & 119 & 94 \\
\hline \multicolumn{5}{|l|}{ Mammalian cell mutagenicity, TPM, } \\
\hline+59 & 101 & 109 & 107 & 104 \\
\hline-59 & 101 & 107 & 107 & 103 \\
\hline
\end{tabular}

Cytotoxicity measured in the neutral red uptake assay. Bacterial mutagenicity in the Salmonella reverse mutation assay and mammalian cell mutagenicity in the mouse lymphoma TK assay. Statistical significances: ${ }^{*}: 0.01<p \leq 0.05,{ }^{* *}: 0.001<p \leq 0.01,{ }^{* * *}: p \leq 0.001$.

under HCI machine smoking conditions was observed, as compared to TPM obtained under ISO smoking conditions, reflecting the higher smoke yields produced under the HCI smoking conditions. With metabolic activation all sensitive tester strains showed decreases in the mutagenic activity per mg TPM, 'tar', or nicotine. The decreases, up to $-45 \%$, were statistically significant for the calculations based on per mg TPM.

\section{In vitro mammalian mutagenicity (ML assay)}

Under ISO machine smoking conditions, dose-dependent increases in TPM mutant frequencies from both the 2R4F and $3 \mathrm{R} 4 \mathrm{~F}$ reference cigarettes were observed, with and without metabolic activation, compared to solvent control. No significant differences in mutagenicity were observed between the reference cigarettes, regardless of the calculation basis. Comparisons of the TPM mutagenicity from the $3 \mathrm{R} 4 \mathrm{~F}$ cigarette by both machine smoking regimens found an increase in total activity of approximately $+150 \%$ per cigarette, with and without metabolic activation. On a per mg TPM basis, statistically significant decreases in activity were observed with $(-34 \%)$ and without $(-23 \%)$ metabolic activation. When expressed on a per mg 'tar' basis, the decreases were less distinct. On a per mg nicotine basis, the activities were not different between both machine smoking regimens.

\section{Ninety-day rat inhalation}

- In life observations and body weights

Following MS-exposure to 2R4F and 3R4F cigarette smoke generated under ISO machine smoking conditions, significant reductions in body weight development in male rats were observed, compared to sham-exposed rats $(\mathrm{P} \leq 0.01)$. In addition, Harderian gland secretion and wet fur were more frequently observed for MS-exposed groups, compared to the sham-exposed groups. The same magnitude of effects was observed for both cigarettes. There were no other significant differences observed between the smoke-exposed groups and sham-exposure groups. 3R4F reference cigarette mainstream smoke was generated under both the ISO and the HCI machine smoking regimens showed the same effect on body weight reduction, Harderian gland secretion, and the occurrence of wet fur.

- Clinical chemistry, hematology and organ weights Following MS-exposure to both the 2R4F and 3R4F cigarettes smoked according to ISO machine smoking conditions, only expected alterations in clinical chemistry (41) were observed (e.g., decreased serum concentrations of proteins, triglycerides, cholesterol), hematology (increased hemoglobin) and organ weights (e.g., decreased thymus weight). There were no further consistent, or significant, differences for these parameters between both reference cigarettes (data not shown). Following exposure to $\mathrm{MS}$ of the $3 \mathrm{R} 4 \mathrm{~F}$ reference cigarette under either machine smoking condition, none of the above parameters was affected by the smoking regimen in a meaningful and consistent way (data not shown).

\section{- Histopathology}

The histopathological evaluation of the respiratory tract organs revealed qualitatively similar findings for the $2 \mathrm{R} 4 \mathrm{~F}$ and $3 \mathrm{R} 4 \mathrm{~F}$ reference cigarettes when smoked under ISO machine smoking conditions that are comparable to expected results following MS exposure reported in the literature (41). Quantitatively, there was no consistent trend (male and female rats, response at various sites) for a difference in toxicity (Table 6), although, numerically, the $3 \mathrm{R} 4 \mathrm{~F}$ reference cigarette smoke-exposed female rats showed effects that were slightly more pronounced than those in the $2 \mathrm{R} 4 \mathrm{~F}$ group.

MS exposure to the $3 \mathrm{R} 4 \mathrm{~F}$ reference cigarette smoked according to ISO and HCI machine smoking conditions did not reveal consistent and meaningful differences. A higher response in the trachea in the male rats exposed to the smoke generated under HCI conditions relative to the ISO groups is due to the fact that at very low incidence rates differences that are small on an absolute basis translate into high relative differences in percent (Table 6, Figure 2). 
Table 5. Relative in vitro toxicity of the 3R4F reference cigarette when smoked according ISO and $\mathrm{HCl}$ machine smoking conditions.

\begin{tabular}{|c|c|c|c|c|}
\hline \multirow{2}{*}{ Assay, measure, smoke fraction } & \multicolumn{4}{|c|}{ 3R4F HCl to 3R4F ISO ratio (\%) } \\
\hline & Per cigarette & Per mg TPM & Per mg 'tar' & Per mg nicotine \\
\hline \multicolumn{5}{|l|}{ Cytotoxicity, $1 / E C_{50}$} \\
\hline TPM & $283^{* * *}$ & $82^{* *}$ & 98 & 106 \\
\hline GVP & $298^{\star * *}$ & 83 & 102 & 112 \\
\hline \multicolumn{5}{|l|}{ Bacterial mutagenicity, TPM, } \\
\hline \multicolumn{5}{|l|}{ Revertants per calculation basis } \\
\hline TA $98, \quad+$ S9 & $267^{* \star *}$ & $75^{\star \star \star}$ & 90 & 99 \\
\hline TA $100,+S 9$ & $258^{\star \star *}$ & $72^{\star \star}$ & 86 & 96 \\
\hline TA1537, +S9 & $198^{\star \star *}$ & $55^{\star *}$ & $66^{*}$ & 74 \\
\hline TA 100, -S9 & $363^{\star \star *}$ & 99 & 120 & 133 \\
\hline \multicolumn{5}{|l|}{ Mammalian cell mutagenicity, TPM, } \\
\hline $1 / C_{3 B}$ & & & & \\
\hline+59 & $242^{* * *}$ & $66^{\star \star *}$ & $79^{* * *}$ & 90 \\
\hline-59 & $276^{\star * *}$ & $77^{* *}$ & 92 & 103 \\
\hline
\end{tabular}

Cytotoxicity measured in the neutral red uptake assay. Bacterial mutagenicity in the Salmonella reverse mutation assay and mammalian cell mutagenicity in the mouse lymphoma TK assay. Statistical significances: ${ }^{*}: 0.01<p \leq 0.05,{ }^{* *}: 0.001<p \leq 0.01,{ }^{* * *}: p \leq 0.001$.

Comparisons performed on the rats after the post-inhalation period did not show differences between the toxicity of the smoke from the $3 \mathrm{R} 4 \mathrm{~F}$ and the $2 \mathrm{R} 4 \mathrm{~F}$ reference cigarettes or between the two smoking regimens (data not shown). With the exception of thymus atrophy, tissues from nonrespiratory organs were not significantly affected by the exposure to cigarette smoke.

Nineteen animals showed histo-morphological alterations that are indicative for a rat respiratory virus (RRV) infection. More sham-exposed rats than MS-exposed rats (16 vs. 3, respectively) were affected. The histopathological evaluation of the lung tissue did not appear to be compromised as the infection-related changes can be discriminated from the morphological alterations associated with MS exposure (2). Therefore, the RRV infections were not considered to interfere with the objective of the study.

\section{DISCUSSION}

In the evaluation of smoke chemistry under ISO machine smoking conditions, similar results for TPM, 'tar', and carbon monoxide were obtained for the $2 \mathrm{R} 4 \mathrm{~F}$ and $3 \mathrm{R} 4 \mathrm{~F}$ reference cigarettes. For some toxicants including the carcinogenic TSNAs and acrylamide, somewhat lower yields were observed for the $3 \mathrm{R} 4 \mathrm{~F}$, compared to the $2 \mathrm{R} 4 \mathrm{~F}$ reference cigarette.

As previously reported from comparisons of the $2 \mathrm{R} 4 \mathrm{~F}$ and the $1 \mathrm{R} 4 \mathrm{~F}$ reference cigarette, some variation in smoke constituent yields could be expected due to year-to-year differences in the tobacco crop (3). Overall, however, the objective to produce a new reference cigarette as close as possible to the previous $2 \mathrm{R} 4 \mathrm{~F}$ reference cigarette was met. Thus, for practical purposes, smoke chemistry data of the $2 \mathrm{R} 4 \mathrm{~F}$ and the $3 \mathrm{R} 4 \mathrm{~F}$ are equivalent. Both cigarettes can be used interchangeably as references, although it is recognized that some smoke constituents as, e.g., acrylamide, NNN, and NNK, show statistically significant differences.

The two reference cigarettes also displayed similar in vitro cytotoxicity and mutagenicity. In addition, there were no meaningful differences in biomarkers or histopathological changes obtained in the inhalation studies in rats that would suggest any significant differences in toxicity from exposure to the smoke of the $3 \mathrm{R} 4 \mathrm{~F}$ reference cigarette, as compared to the $2 \mathrm{R} 4 \mathrm{~F}$ reference cigarette. This also holds true when the $3 \mathrm{R} 4 \mathrm{~F}$ data are compared to published data for the $2 \mathrm{R} 4 \mathrm{~F}$ reference cigarette $(2,8,15)$. Accordingly, the toxicological profiles of the $2 \mathrm{R} 4 \mathrm{~F}$ and the $3 \mathrm{R} 4 \mathrm{~F}$ reference cigarettes are considered to be equivalent.

As expected e.g. $(7,8)$, due to the significantly higher amount of 3R4F smoke generated under the intense HCI machine smoking conditions, when expressed on a per cigarette basis, toxicants yields, in vitro mutagenicity and cytotoxicity, and inhalation toxicity increased compared to when smoked according to ISO machine smoking conditions.

However, as with other cigarettes, comparisons on a per mg TPM, per mg 'tar', or per mg nicotine basis, were reduced under the HCI machine smoking conditions. This observation has been previously reported or can be deduced from data presented in similar investigations of machine smoking protocols for other cigarettes $(8,9,42,43)$. One of the potential explanations for this seemingly counterintuitive observation is based on the increase in the flow of air and thus oxygen that is drawn through the burning zone of the cigarette. Specifically, the higher puff volume that, due to occluded filter ventilation, cannot bypass the burning zone of the cigarette, resulting in higher oxygen supply to the burning zone and thus to higher combustion temperatures when smoked under HCI machine smoking conditions. It can be hypothesized that higher combustion temperatures obtained under the HCI machine smoking conditions might result in more complete combustion and thus to a lower yield of cytotoxic and mutagenic toxicants. This is consistent with the increase in water in the TPM under intense machine smoking conditions (8).

It is noteworthy that the same authors have demonstrated that these reductions in toxicant yields and cytotoxic and mutagenic activity under intense machine smoking conditions, when normalized to TPM, 'tar', or nicotine, are less apparent between different cigarettes. Differences observable for different cigarettes under ISO machine smoking conditions are also not as apparent when smoked 
Table 6. Relative in vivo toxicity of the $3 \mathrm{R} 4 \mathrm{~F}$ versus the $2 \mathrm{R} 4 \mathrm{~F}$ reference cigarette when smoked according to ISO machine smoking conditions, and the 3R4F reference cigarette when smoked under both the $\mathrm{HCl}$ and ISO machine smoking conditions.

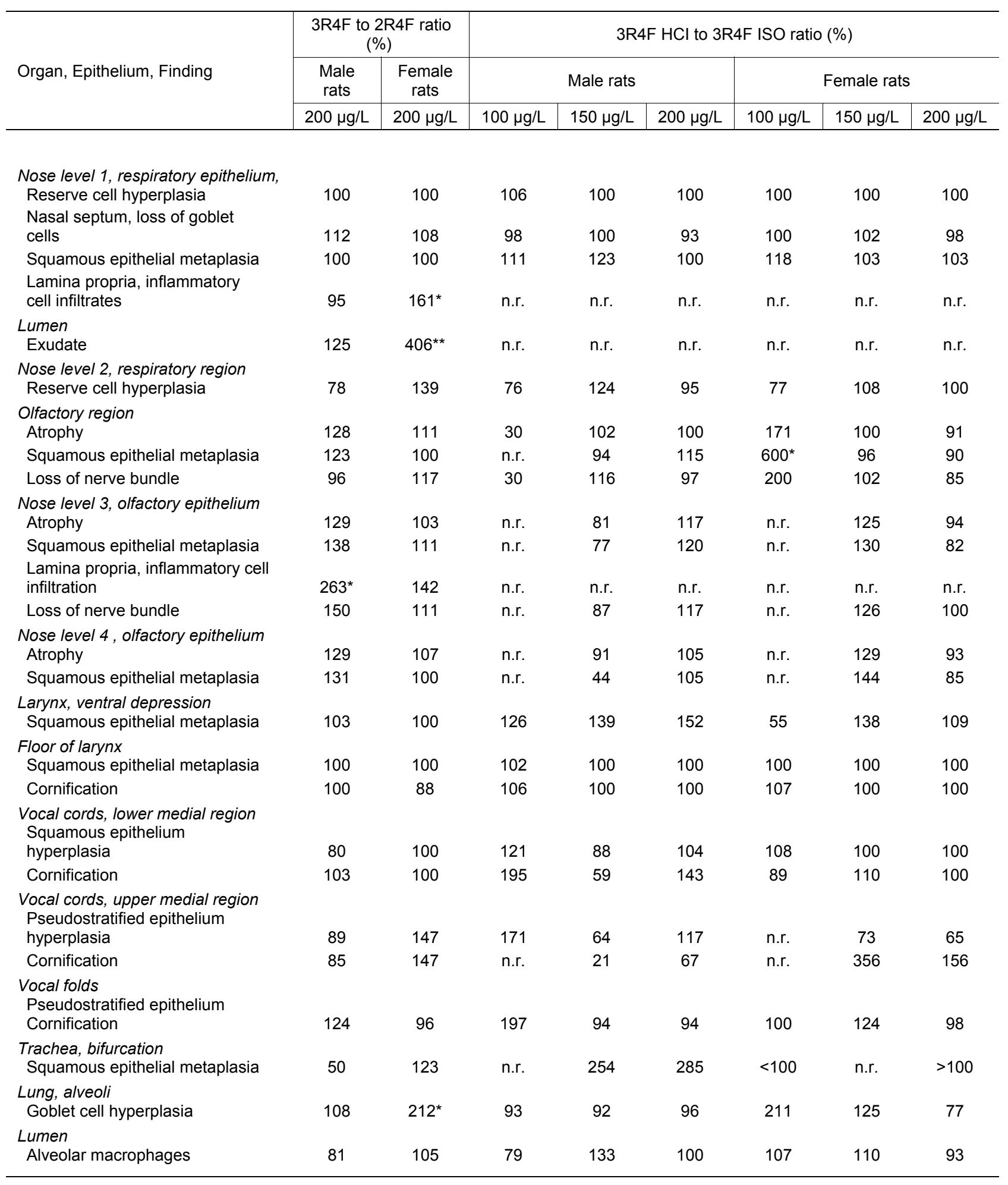

Values are derived from mean scores for histopathological changes after a 90 -day inhalation period. Statistical significances: * $0.01<p \leq 0.05$, ${ }^{* *}: 0.001<p \leq 0.01,{ }^{* * *}: p \leq 0.001$, n.r.: not recorded.

under more intense machine smoking regimens (44). While notable effects were observed between different machine smoking conditions for smoke chemistry and in vitro assessments, there were no such differences noted in the in vivo investigations. The results are qualitatively identical to previous results observed in smoke-exposed rats $(2,15,41$, $45,46)$. There were also no quantitative differences in toxic effects detected in our study between both machine smoking conditions. Recently, distinct differences have been published regarding the in vivo toxicity of cigarette smoke generated under smoking regimens that were rather extreme in intensity (8). It can be argued that the in vivo endpoints were different from those reported here. The study published by ROEMER et al. deals with dermal 
tumorigenicity (skin painting) in mice and not with irritative effects as presented in this publication. Due to their study design (2-stage protocol: mice initiated by dermal application of a carcinogen and subsequent application of cigarette smoke condensate) they put their emphasis of their assessment on the promoting activity (47) of cigarette smoke condensate. However, the promoting, non-genotoxic activity of cigarette smoke has been linked to its irritative capacity (48). As such, one might expect similarities in response between effects in the inhalation toxicity and the 2-stage dermal tumorigenicity study. This apparent discrepancy might be explainable by the fact, that by inhalation exposure both, the particulate as well as the gas phase constituents can exert their action. The mouse skin painting assay, in contrast, investigates only the particulate phase of cigarette smoke. Further research on the possible differences behavior of the gas/vapor phase and the particulate phase under different intensities of smoking regimens might contribute to the understanding of the inherent toxicity of cigarette smoke.

There were two exposure regimens, i.e., seven days per week at $200 \mu \mathrm{g} / \mathrm{L}$ TPM and five days per week at 100, 150, and $200 \mu \mathrm{g} / \mathrm{L}$, all with a daily exposure duration of six hours. The smoke induced effects were for the same exposure concentration of $200 \mu \mathrm{g} / \mathrm{L}$ quantitatively and even qualitatively approximately the same. The group with 5 days exposure scored, as a mean, 0.6 points lower than the group with 7 days per week. The group with $150 \mu \mathrm{g} / \mathrm{L}$ and 5 days per week scored slightly lower, i.e., 0.8 points. This can be interpreted that the toxicity may be somewhat more dependent on the concentration than on the weekly exposure time, which is in line with results obtained for 21 inhalation toxicants (49) or with cigarette smoke (50). However, both exposure regimens, i.e., 7 days or 5 days per week, are obviously suitable methods to assess the toxicity of cigarette smoke.

Expressing smoke chemistry or toxicity data on a calculation basis other than on a per cigarette basis, i.e., a normalization to account for different yield data, as it is performed here for the comparison of the ISO and HCI machine smoking conditions, always needs to be interpreted cautiously due to nonlinear relationships and possibly unidentified measurement biases. Both complications are especially important for low delivery cigarette data (51). Nevertheless, interpretations of normalized data using all bases of calculation are useful for evaluating human exposure, even for cigarettes with low smoke deliveries, as they allow at least a meaningful estimate of the amount of toxicants or biological activity accompanied with a certain amount of nicotine, 'tar', etc. For the 2R4F and 3R4F reference cigarette data presented here, these precautions do not apply, as both cigarettes have high yields under machine smoking conditions. Standardized machine smoking conditions that apply either more or less intense smoking parameters can provide only one specific combination of possible settings of characteristics, such as fixed puff volume, puff duration, and puff frequency, and are not meant to mimic human smoking behavior, nor could they be expected to do so, as each smoker smokes differently. Accordingly, there is no typical human smoker and no typical human smoking regimen (18-20). However, the results obtained under different

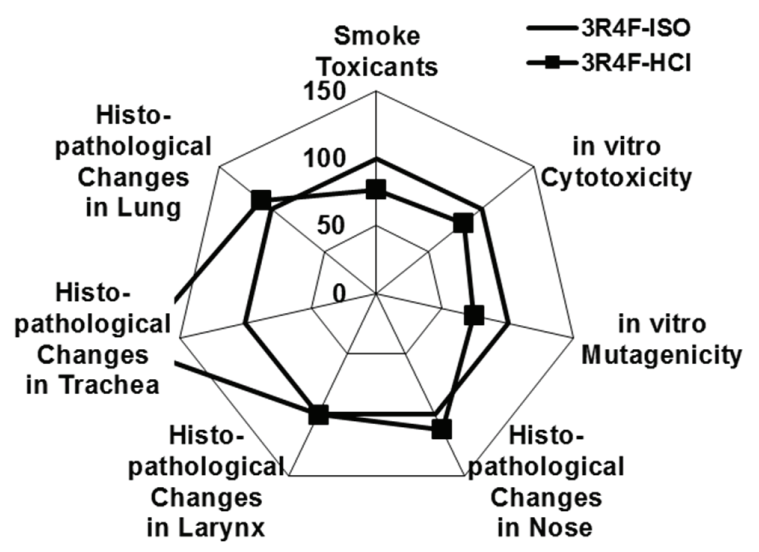

Figure 2. Summary of comparisons of the 3R4F reference cigarettes $\mathrm{HCl}$ to ISO machine smoking conditions - means from Tables 3,5 , and 6 .

machine smoking conditions of different intensity can give insight into the possible spectrum of different smoke qualities and the underlying mechanisms.

\section{CONCLUSIONS}

Overall, the results of the present study suggest equivalent smoke chemistry and toxicity for the $3 \mathrm{R} 4 \mathrm{~F}$ and $2 \mathrm{R} 4 \mathrm{~F}$ reference cigarettes when smoked under the same smoking regimen. As observed already for other cigarettes, 3R4F mainstream smoke generated under intense smoking conditions is generally less cytotoxic and mutagenic in vitro than the smoke generated under less intense conditions. The in vivo inhalation toxicity, however, seems not to be different.

\section{ACKNOWLEDGEMENT}

The authors are grateful to the staff at Philip Morris Research Laboratories in Cologne, Germany, and Leuven, Belgium, for the excellent technical performance of the studies described.

\section{REFERENCES}

1. Chepiga, T.A., M.J. Morton, P.A. Murphy, J.T. Avalos, B.R. Bombick, D.J. Doolittle, M.F. Borgerding, and J.E. Swauger: A comparison of the mainstream smoke chemistry and mutagenicity of a representative sample of the US cigarette market with two Kentucky reference cigarettes (K1R4F and K1R5F); Food Chem. Toxicol. 38 (2000) 949-962.

2. Werley, M.S., S.A. Freelin, S.E. Wrenn, B. Gerstenberg, E. Roemer, H. Schramke, E. Van Miert, P. Vanscheeuwijck, S. Weber, and C.R. Coggins: Smoke chemistry, in vitro and in vivo toxicology evaluations of the electrically heated cigarette smoking system series K; Regul. Toxicol. Pharmacol. 52 (2008) 122-139.

3. Chen, P.X. and S.C. Moldoveanu: Mainstream smoke 
chemical analyses for 2R4F Kentucky reference cigarette; Beitr. Tabakforsch. Int. 20 (2003) 448-458.

4. Counts, M.E., F.S. Hsu, and F.J. Tewes: Development of a commercial cigarette "market map" comparison methodology for evaluating new or non-conventional cigarettes; Regul. Toxicol. Pharmacol. 46 (2006) 225-242.

5. Adam, T., S. Mitschke, T. Streibel, R.R. Baker, and R. Zimmermann: Puff-by-puff resolved characterisation of cigarette mainstream smoke by single photon ionisation (SPI)-time-of-flight mass spectrometry (TOFMS): comparison of the $2 \mathrm{R} 4 \mathrm{~F}$ research cigarette and pure Burley, Virginia, Oriental and Maryland tobacco cigarettes; Anal. Chim. Acta 572 (2006) 219-229.

6. Intorp, M., S. Purkis, M. Whittaker, and W. Wright: Determination of "Hoffmann Analytes" in Cigarette Mainstream Smoke. The Coresta 2006 Joint Experiment; Beitr. Tabakforsch. Int. 23 (2009) 161-202.

7. Rickert, W.S., A.H. Trivedi, R.A. Momin, W.G. Wright, and J.H. Lauterbach: Effect of smoking conditions and methods of collection on the mutagenicity and cytotoxicity of cigarette mainstream smoke; Toxicol. Sci. 96 (2007) 285-293.

8. Roemer, E., T.H. Ottmueller, V. Zenzen, S. Wittke, F. Radtke, I. Blanco, and R.A. Carchman: Cytotoxicity, mutagenicity, and tumorigenicity of mainstream smoke from three reference cigarettes machine-smoked to the same yields of total particulate matter per cigarette; Food Chem. Toxicol. 47 (2009) 1810-1818.

9. Roemer, E., R. Stabbert, K. Rustemeier, D.J. Veltel, T.J. Meisgen, W. Reininghaus, R.A. Carchman, C.L. Gaworski, and K.F. Podraza: Chemical composition, cytotoxicity and mutagenicity of smoke from US commercial and reference cigarettes smoked under two sets of machine smoking conditions; Toxicology 195 (2004) 31-52.

10. Schramke, H., T.J. Meisgen, F.J. Tewes, W. Gomm, and E. Roemer: The mouse lymphoma thymidine kinase assay for the assessment and comparison of the mutagenic activity of cigarette mainstream smoke particulate phase; Toxicology 227 (2006) 193-210.

11. Chen, J., R. Higby, D. Tian, D. Tan, M.D. Johnson, Y. Xiao, K.J. Kellar, S. Feng, and P.G. Shields: Toxicological analysis of low-nicotine and nicotine-free cigarettes; Toxicology 249 (2008) 194-203.

12. Gaworski, C.L., R. Lemus-Olalde, and E.L. Carmines: Toxicological evaluation of potassium sorbate added to cigarette tobacco; Food Chem. Toxicol. 46 (2008) 339-351.

13. Newland, N. and A. Richter: Agents associated with lung inflammation induce similar responses in NCI-H292 lung epithelial cells; Toxicol. in vitro 22 (2008) 1782-1728.

14. Carter, C.A. and J.T. Hamm: Multiplexed quantitative high content screening reveals that cigarette smoke condensate induces changes in cell structure and function through alterations in cell signaling pathways in human bronchial cells; Toxicology 261 (2009) 89-102.

15. Higuchi, M.A., J. Sagartz, W.K. Shreve, and P.H. Ayres: Comparative subchronic inhalation study of smoke from the $1 \mathrm{R} 4 \mathrm{~F}$ and $2 \mathrm{R} 4 \mathrm{~F}$ reference cigarettes; Inhal. Toxicol. 16 (2004) 1-20.

16. ISO 3308: Routine analytical cigarette smoking machine - Definitions and standard conditions. International Organization for Standardization, Geneva, Switzerland, 2000.

17. Health Canada: Determination of 'tar', nicotine and carbon monoxide in mainstream smoke. Health Canada - Official Method 1999.

18. Marian, C., R.J. O'Connor, M.V. Djordjevic, V.W. Rees, D.K. Hatsukami, and P.G. Shields: Reconciling human smoking behavior and machine smoking patterns: implications for understanding smoking behavior and the impact on laboratory studies; Cancer Epidemiol. Biomarkers Prev. 18 (2009) 3305-3320.

19. Hammond, D., G.T. Fong, K.M. Cummings, and A. Hyland: Smoking topography, brand switching, and nicotine delivery: results from an in vivo study; Cancer Epidemiol. Biomarkers Prev. 14 (2005) 1370-1375.

20. Hammond, D., G.T. Fong, K.M. Cummings, R.J. O'Connor, G.A. Giovino, and A. McNeill: Cigarette yields and human exposure: a comparison of alternative testing regimens; Cancer Epidemiol. Biomarkers Prev. 15 (2006) 1495-1501.

21. ISO 3402: Tobacco and tobacco products - Atmosphere for conditioning and testing. International Organization for Standardization, Geneva, Switzerland, 1999.

22. Radtke, F., M. Schmickler, W. Ochel, and H. Schaffernicht: Pumpenanordnung. Patent EP1832745 (A1) In Office, E.P. (ed.), Germany, 2007.

23. ISO 4387: Determination of total and nicotine-free dry particulate matter using routine analytical smoking machine. International Organization for Standardization, Geneva, Switzerland, 1991.

24. ISO 10315: Cigarettes - Determination of nicotine in smoke condensate - Gas chromatographic method (2nd ed.). International Organization for Standardization, Geneva, Switzerland, 2000.

25. ISO 8454: Cigarettes - Determination of carbon monoxide in the vapour phase of cigarette smoke NDIR method (3rd ed.). International Organization for Standardization, Geneva, Switzerland, 2007.

26. CORESTA: Recommended Method No. 74 Determination of selected carbonyls in mainstream cigarette smoke by high performance liquid chromatography (HPLC). Cooperation Centre for Scientific Research Relative to Tobacco, 2011.

27. CORESTA: Recommended Method No. 70 Determination of selected volatile organic compounds in the mainstream smoke of cigarettes - gas chromatography-mass spectrometry method. Co-operation Centre for Scientific Research Relative to Tobacco, 2010.

28. Diekmann, J., A. Wittig, and R. Stabbert: Gas chromatographic-mass spectrometric analysis of acrylamide and acetamide in cigarette mainstream smoke after on-column injection; J. Chromatogr. Sci. 46 (2008) 659-663.

29. Diekmann, J., M. Douda, and K. Rustemeier: Rapid and sensitive method for the determination of propylene oxide in cigarette mainstream smoke by gas chromatography-mass spectrometry; J. Chromatogr. Sci. 44 
(2006) 32-34.

30. Stabbert, R., K.H. Schaefer, C. Biefel, and K. Rustemeier: Analysis of aromatic amines in cigarette smoke; Rapid Commun. Mass. Spectrom. 17 (2003) 2125-2132.

31. CORESTA: Recommended Method (3rd Draft): The determination of nitric oxide in mainstream smoke of cigarettes by chemiluminescent analysis; Available at (accessed January 2012): http://legacy.library.ucsf.edu/tid/vpe43d00/pdf; jsessionid=BEB3E72646 C743124370F7007FA332D8.tobacco03.

32. Mottier, N. and F. Jeanneret: Evaluation of two derivatization reagents for the determination by LC-MS/MS of ammonia in cigarette mainstream smoke; J. Agric. Food Chem. 59 (2011) 92-97.

33. Wagner, K.A., N.H. Finkel, J.E. Fossett, and I.G. Gillman: Development of a quantitative method for the analysis of tobacco-specific nitrosamines in mainstream cigarette smoke using isotope dilution liquid chromatography/electrospray ionization tandem mass spectrometry; Anal. Chem. 77 (2005) 1001-1006.

34. Maron, D.M. and B.N. Ames: Revised methods for the Salmonella mutagenicity test; Mutat. Res. 113 (1983) 173-215.

35. Cole, J., W.J. Muriel, and B.A. Bridges: The mutagenicity of sodium fluoride to L5178Y [wild-type and $\mathrm{TK}+/-$ (3.7.2c)] mouse lymphoma cells; Mutagenesis 1 (1986) 157-167.

36. Gaworski, C.L., H. Schramke, J. Diekmann, T.J. Meisgen, F.J. Tewes, D.J. Veltel, P.M. Vanscheeuwijck, N. Rajendran, M. Muzzio, and H.J. Haussmann: Effect of filtration by activated charcoal on the toxicological activity of cigarette mainstream smoke from experimental cigarettes; Inhal. Toxicol. 21 (2009) 688-704.

37. ISO 3308: Routine analytical cigarette-smoking machine - Definitions and standard conditions; International Organization for Standardization, Geneva, Switzerland, 2000.

38. Young, J.T.: Histopathologic examination of the rat nasal cavity; Fundam. Appl. Toxicol. 1 (1981) 309-312.

39. Lewis, D.J.: Factors affecting the distribution of tobacco smoke-induced lesions in the rodent larynx; Toxicol. Lett. 9 (1981) 189-194.

40. Carmines, E.L., C.L. Gaworski, A.S. Faqi, and N. Rajendran: In utero exposure to $1 \mathrm{R} 4 \mathrm{~F}$ reference cigarette smoke: evaluation of developmental toxicity; Toxicol. Sci. 75 (2003) 134-147.

41. Vanscheeuwijck, P.M., A. Teredesai, P.M. Terpstra, J. Verbeeck, P. Kuhl, B. Gerstenberg, S. Gebel, and E.L. Carmines: Evaluation of the potential effects of ingredients added to cigarettes. Part 4: subchronic inhalation toxicity; Food Chem. Toxicol. 40 (2002) 113-131.
42. Counts, M.E., M.J. Morton, S.W. Laffoon, R.H. Cox, and P.J. Lipowicz: Smoke composition and predicting relationships for international commercial cigarettes smoked with three machine-smoking conditions; Regul. Toxicol. Pharmacol. 41 (2005) 185-227.

43. Okuwa, K., M. Tanaka, Y. Fukano, H. Nara, Y. Nishijima, and T. Nishino: In vitro micronucleus assay for cigarette smoke using a whole smoke exposure system: a comparison of smoking regimens; Exp. Toxicol. Pathol. 62 (2009) 433-40.

44. Roemer, E. and R.A. Carchman: Limitations of cigarette machine smoking regimens; Toxicol. Lett. 203 (2011) 20-27.

45. Terpstra, P.M., A. Teredesai, P.M. Vanscheeuwijck, J.Verbeeck, G. Schepers, F. Radtke, P. Kuhl, W. Gomm, E. Anskeit, and G. Patskan: Toxicological evaluation of an electrically heated cigarette. Part 4: Subchronic inhalation toxicology; J. Appl. Toxicol. 23 (2003) 349-362.

46. Baker, R.R., E.D. Massey, and G. Smith: An overview of the effects of tobacco ingredients on smoke chemistry and toxicity; Food Chem. Toxicol. 42 Suppl. (2004) S53-S83.

47. DiGiovanni, J.: Multistage carcinogenesis in mouse skin; Pharmacol. Ther. 54 (1992) 63-128.

48. Berenblum, I.: The cocarcinogenic action of croton resin; Cancer Res. 1 (1941) 44-48.

49. Belkebir, E., C. Rousselle, C. Duboudin, L. Bodin, and N. Bonvallot: Haber's rule duration adjustments should not be used systematically for risk assessment in public health decision-making; Toxicol. Lett. 204 (2010) $148-155$.

50. Tsuji, H., K.M. Lee, K. Yoshino, H. Nakamura, G. Lulham, R. Renne, and H. Yoshimura: Comparison of the physiological and morphological effects of cigarette smoke exposure at comparable weekly doses on Sprague-Dawley rats; Inhal. Toxicol. 23 (2010) 17-32.

51. St Charles, F.K., C.J. Cook, and P.M. Clayton: The linear relationship between cigarette 'tar' and nicotine yields: Regulatory implications for smoke constituent ratios; Regul. Toxicol. Pharmacol. 59 (2011) 143-148.

Corresponding author:

Dr. Ewald Roemer

c/o Philip Morris Intl.

Rue des Usines 90

2000 Neuchâtel

Switzerland

ewald.roemer@pmi.com 
Table A. Smoke constituent yields for the $3 R 4 \mathrm{~F}$ and the $2 \mathrm{R} 4 \mathrm{~F}$ reference cigarettes.

\begin{tabular}{|c|c|c|c|c|c|c|c|}
\hline \multirow[t]{2}{*}{ Analyte } & \multirow{2}{*}{$\begin{array}{l}\text { Unit } \\
\text { per cig }\end{array}$} & \multicolumn{2}{|c|}{$\begin{array}{c}\text { 3R4F } \\
\text { ISO }\end{array}$} & \multicolumn{2}{|c|}{$\begin{array}{c}\text { 2R4F } \\
\text { ISO }\end{array}$} & \multicolumn{2}{|c|}{$\begin{array}{c}3 \mathrm{R} 4 \mathrm{~F} \\
\mathrm{HCl}\end{array}$} \\
\hline & & $M$ & SE & $M$ & SE & $M$ & SE \\
\hline \multicolumn{8}{|l|}{ ISO Parameter } \\
\hline TPM & $\mathrm{mg}$ & 9.77 & 0.04 & 9.32 & 0.08 & 37.7 & 0.3 \\
\hline 'Tar' & $\mathrm{mg}$ & 7.98 & 0.03 & 7.57 & 0.05 & 25.5 & 0.2 \\
\hline Nicotine & $\mathrm{mg}$ & 0.707 & 0.005 & 0.678 & 0.007 & 1.90 & 0.02 \\
\hline Water & $\mathrm{mg}$ & 1.08 & 0.04 & 1.08 & 0.02 & 10.3 & 0.2 \\
\hline $\mathrm{CO}$ & $\mathrm{mg}$ & 11.2 & 0.1 & 10.7 & 0.1 & 32.7 & 0.2 \\
\hline \multicolumn{8}{|l|}{ Aldehydes } \\
\hline Formaldehyde & $\mu \mathrm{g}$ & 20.0 & 0.7 & 18.4 & 0.4 & 68.1 & 1.4 \\
\hline Acetaldehyde & $\mu \mathrm{g}$ & 567 & 10 & 542 & 5 & 1534 & 32 \\
\hline Acrolein & $\mu g$ & 56.7 & 1.4 & 54.7 & 0.7 & 155 & 3 \\
\hline Propionaldehyde & $\mu \mathrm{g}$ & 48.4 & 0.9 & 47.0 & 0.4 & 124 & 3 \\
\hline Crotonaldehyde & $\mu \mathrm{g}$ & 10.1 & 0.3 & 9.00 & 0.21 & 43.1 & 0.9 \\
\hline \multicolumn{8}{|l|}{ Aliphatic dienes } \\
\hline 1,3-Butadiene & $\mu g$ & 38.5 & 1.2 & 38.9 & 1.6 & 76.5 & 2.1 \\
\hline Isoprene & $\mu \mathrm{g}$ & 395 & 11 & 411 & 5 & 863 & 27 \\
\hline \multicolumn{8}{|l|}{ Acid derivatives } \\
\hline Acetamide & $\mu g$ & 4.46 & 0.12 & 4.37 & 0.05 & 15.2 & 0.2 \\
\hline Acrylamide & $\mu \mathrm{g}$ & 1.37 & 0.02 & 1.57 & 0.03 & - & - \\
\hline Acrylonitrile & $\mu \mathrm{g}$ & 26.4 & 0.7 & 26.5 & 0.5 & 67.0 & 0.8 \\
\hline \multicolumn{8}{|l|}{ Epoxides } \\
\hline Ethylene oxide & $\mu g$ & 9.24 & 0.18 & 9.56 & 0.36 & - & - \\
\hline \multicolumn{8}{|l|}{ Nitro compounds } \\
\hline 2-Nitropropane & $\mu \mathrm{g}$ & 18.3 & 0.3 & 20.4 & 1.7 & 35.9 & 0.6 \\
\hline \multicolumn{8}{|l|}{ Aromatic amines } \\
\hline o-Toluidine & $\mathrm{ng}$ & 54.1 & 0.7 & 53.5 & 0.7 & 99.3 & 1.4 \\
\hline o-Anisidine & $\mathrm{ng}$ & 2.32 & 0.04 & 2.23 & 0.03 & 4.25 & 0.12 \\
\hline 2-Naphthylamine & $\mathrm{ng}$ & 5.69 & 0.07 & 5.83 & 0.12 & 10.1 & 0.4 \\
\hline 4-Aminobiphenyl & $\mathrm{ng}$ & 1.01 & 0.01 & 1.04 & 0.02 & 2.24 & 0.06 \\
\hline \multicolumn{8}{|l|}{ Halogen compounds } \\
\hline Vinyl chloride & $\mathrm{ng}$ & 49.7 & 2.0 & 57.2 & 2.1 & 69.7 & 2.1 \\
\hline \multicolumn{8}{|l|}{ Inorganic compounds } \\
\hline Nitrogen oxides & $\mu g$ & 265 & 3 & 270 & 5 & 626 & 6 \\
\hline Hydrogen cyanide & $\mu g$ & 70.9 & 1.9 & 69.8 & 1.5 & 319 & 9 \\
\hline Ammonia & $\mu \mathrm{g}$ & 11.1 & 0.2 & 12.4 & 0.2 & - & - \\
\hline \multicolumn{8}{|c|}{ Monocyclic aromatic hydrocarbons } \\
\hline Benzene & $\mu g$ & 45.7 & 0.9 & 46.6 & 0.6 & 104 & 1 \\
\hline Toluene & $\mu \mathrm{g}$ & 73.6 & 1.4 & 73.7 & 1.0 & 208 & 5 \\
\hline Styrene & $\mu \mathrm{g}$ & 6.00 & 0.22 & 6.09 & 0.09 & 24.9 & 0.9 \\
\hline \multicolumn{8}{|c|}{ Volatile $N$-nitrosamines } \\
\hline NDMA & $\mathrm{ng}$ & $<5.00$ & - & $<5.00$ & - & $<10.0$ & - \\
\hline NMEA & ng & $<10.0$ & - & $<10.0$ & - & $<20.0$ & - \\
\hline NDEA & ng & $<7.00$ & - & $<7.00$ & - & $<14.0$ & - \\
\hline NPRA & $\mathrm{ng}$ & $<11.0$ & - & $<11.0$ & - & $<22.0$ & - \\
\hline NBUA & ng & $<9.00$ & - & $<9.00$ & - & $<18.0$ & - \\
\hline NPY & $\mathrm{ng}$ & $<7.00$ & - & $<7.00$ & - & $<14.0$ & - \\
\hline NPI & $\mathrm{ng}$ & $<8.00$ & - & $<8.00$ & - & $<16.0$ & - \\
\hline \multicolumn{8}{|c|}{ Tobacco-specific N-nitrosamines } \\
\hline NNN & $\mathrm{ng}$ & 92.1 & 1.5 & 110 & 2 & 276 & 3 \\
\hline NNK & ng & 85.5 & 1.8 & 110 & 2 & 243 & 6 \\
\hline NAB & $\mathrm{ng}$ & 9.60 & 0.46 & 9.33 & 0.40 & 24.0 & 0.5 \\
\hline NAT & ng & 92.9 & 4.4 & 87.9 & 3.6 & 251 & 5 \\
\hline
\end{tabular}


Table A. (cont.).

\begin{tabular}{|c|c|c|c|c|c|c|c|}
\hline \multirow[t]{2}{*}{ Analyte } & \multirow{2}{*}{$\begin{array}{l}\text { Unit } \\
\text { per cig }\end{array}$} & \multicolumn{2}{|c|}{$\begin{array}{l}\text { 3R4F } \\
\text { ISO }\end{array}$} & \multicolumn{2}{|c|}{$\begin{array}{c}\text { 2R4F } \\
\text { ISO }\end{array}$} & \multicolumn{2}{|c|}{$\begin{array}{c}3 \mathrm{R} 4 \mathrm{~F} \\
\mathrm{HCl}\end{array}$} \\
\hline & & $\mathrm{M}$ & SE & $\mathrm{M}$ & SE & $\mathrm{M}$ & SE \\
\hline \multicolumn{8}{|l|}{ Phenols } \\
\hline Phenol & $\mu \mathrm{g}$ & 7.04 & 0.10 & 6.52 & 0.13 & 14.8 & 0.3 \\
\hline Catenol & $\mu \mathrm{g}$ & 37.1 & 0.2 & 36.2 & 0.3 & 89.3 & 0.5 \\
\hline Hydroquinone & $\mu \mathrm{g}$ & 29.1 & 0.1 & 27.4 & 0.2 & 75.7 & 1.0 \\
\hline \multicolumn{8}{|c|}{ Polycyclic aromatic hydrocarbons } \\
\hline Pyrene & $\mathrm{ng}$ & 38.0 & 0.8 & 35.3 & 0.2 & 92.5 & 1.9 \\
\hline Benz[a]anthracene & $\mathrm{ng}$ & 11.8 & 0.3 & 11.2 & 0.1 & 29.8 & 0.6 \\
\hline Benzo $[b]$ fluoranthene & $\mathrm{ng}$ & 5.09 & 0.08 & 4.52 & 0.04 & 13.2 & 0.3 \\
\hline Benzo[j]fluoranthene & $\mathrm{ng}$ & 3.24 & 0.07 & 2.86 & 0.04 & 8.35 & 0.19 \\
\hline Benzo[k]fluoranthene & $\mathrm{ng}$ & 2.02 & 0.05 & 1.74 & 0.02 & 5.38 & 0.11 \\
\hline Benzo[a]pyrene & $\mathrm{ng}$ & 6.73 & 0.11 & 6.21 & 0.07 & 16.2 & 0.4 \\
\hline Dibenz $[a, h]$ anthracene & $\mathrm{ng}$ & $<0.970$ & - & $<0.970$ & - & $<2.42$ & - \\
\hline Dibenzo[a,e]pyrene & $\mathrm{ng}$ & 0.173 & 0.005 & 0.160 & 0.004 & 0.858 & 0.015 \\
\hline Dibenzo[a, $h$ ]pyrene & ng & $<0.230$ & - & $<0.230$ & - & $<0.575$ & - \\
\hline Dibenzo[a,i]pyrene & $\mathrm{ng}$ & $<0.220$ & - & $<0.220$ & - & $<0.550$ & - \\
\hline Dibenzo[a,/]pyrene & $\mathrm{ng}$ & $<0.190$ & - & $<0.190$ & - & $<0.475$ & - \\
\hline Indeno[1,2,3-cd]pyrene & ng & 2.87 & 0.04 & 2.55 & 0.02 & 7.37 & 0.10 \\
\hline 5-Methylchrysene & $\mathrm{ng}$ & $<0.400$ & - & $<0.400$ & - & $<1.00$ & - \\
\hline \multicolumn{8}{|l|}{ Elements } \\
\hline Cadmium & $\mathrm{ng}$ & 38.3 & 0.3 & 38.5 & 1.2 & 146 & 3 \\
\hline Chromium & $\mathrm{ng}$ & 2.48 & 0.09 & 2.36 & 0.12 & $<6.40$ & - \\
\hline Nickel & $\mathrm{ng}$ & $<2.10$ & - & $<2.10$ & - & $<8.40$ & - \\
\hline Lead & $\mathrm{ng}$ & 9.89 & 0.13 & 10.6 & 0.1 & 32.2 & 1.1 \\
\hline Arsenic & ng & 2.81 & 0.01 & 2.58 & 0.01 & 8.62 & 0.38 \\
\hline Selenium & $\mathrm{ng}$ & 0.621 & 0.018 & 0.606 & 0.017 & - & - \\
\hline Mercury & $\mathrm{ng}$ & 2.81 & 0.1 & 3.13 & 0.15 & - & - \\
\hline
\end{tabular}

$\mathrm{CO}=$ carbon monoxide; $\mathrm{LOQ}=$ at least one value below limit of quantification; NBUA $=N$-nitrosodi-n-butylamine; NDMA $=N$ nitrosodimethylamine; NDEA $=N$-nitroso-n-diethylamine; NMEA $=N$-methylethanolamine; NNN $=N^{\prime}$-nitrosonornicotine; NNK $=4$ (methylnitrosamino)-1-(3-pyridyl)-1-butanone; NPI = N-nitrosopiperidine; NPRA = N-nitrosodi-n-propylamine; NPY = N-nitrosopyrrolidine; TPM $=$ total particulate matter.

The 3R4F reference cigarette values obtained under $\mathrm{HCl}$ machine-smoking conditions (3R4F-HCl) were derived in separate studies to the 2R4F (2R4F-ISO) and 3R4F (3R4F-ISO) values obtained under ISO machine smoking conditions. The calculation of 3R4F-HCI/3R4F-ISO ratios may result in slightly different values as presented in Tables 3, 5, and 6 as concurrently determined 3RF4-ISO values (not presented) are used.

Table B. In vitro toxicity of the $3 \mathrm{R} 4 \mathrm{~F}$ and the $2 \mathrm{R} 4 \mathrm{~F}$ reference cigarettes.

\begin{tabular}{|c|c|c|c|c|c|c|c|}
\hline \multirow[t]{2}{*}{ Assay, measure, smoke fraction } & \multirow[t]{2}{*}{ Unit } & \multicolumn{2}{|c|}{$\begin{array}{l}3 \mathrm{R} 4 \mathrm{~F} \\
\text { ISO }\end{array}$} & \multicolumn{2}{|c|}{$\begin{array}{l}\text { 2R4F } \\
\text { ISO }\end{array}$} & \multicolumn{2}{|c|}{$\begin{array}{c}3 \mathrm{R} 4 \mathrm{~F} \\
\mathrm{HCl}\end{array}$} \\
\hline & & $\mathrm{M}$ & SE & $\mathrm{M}$ & SE & M & SE \\
\hline Cytotoxicity, 1/EC $C_{50}$ & $\mathrm{~mL} / \mathrm{mg}$ TPM & & & & & & \\
\hline TPM & & 10.3 & 0.2 & 10.6 & 0.4 & 8.6 & 0.3 \\
\hline GVP & & 7.8 & 0.3 & 8.4 & 0.3 & 6.2 & 0.3 \\
\hline Bacterial mutagenicity, slope, TPM & Revertants/mg TPM & & & & & & \\
\hline $\mathrm{TA} 98, \quad+\mathrm{S} 9$ & & 2343 & 85 & 2507 & 52 & 1991 & 116 \\
\hline TA 100, +S9 & & 1285 & 72 & 1232 & 106 & 986 & 74 \\
\hline TA1537, +S9 & & 395 & 34 & 337 & 43 & 281 & 24 \\
\hline TA 100, $\quad-S 9$ & & 100 & 17 & 83 & 19 & 130 & 25 \\
\hline $\begin{array}{l}\text { Mammalian cell mutagenicity, } 1 / C_{3 B} \text {, } \\
\text { TPM, }\end{array}$ & $\mathrm{mL} / \mathrm{mg}$ TPM & & & & & & \\
\hline +S9 & & 6.3 & 0.3 & 5.8 & 0.3 & 4.1 & 0.5 \\
\hline -S9 & & 27.7 & 2.9 & 26.0 & 1.8 & 23.2 & 1.6 \\
\hline
\end{tabular}

Cytotoxicity measured in the neutral red uptake assay. Bacterial mutagenicity in the Salmonella reverse mutation assay and mammalian cell mutagenicity in the mouse lymphoma TK assay. The 3R4F reference cigarette values obtained were obtained under $\mathrm{HCl}$ machine smoking conditions (3R4F-HCl). 3R4F (3R4F-ISO) and 2R4F (2R4F-ISO) values obtained under ISO machine smoking conditions. Three replicates per assay in the cytotoxicity and two in the bacterial and mammalian mutagenicity assay. $\mathrm{M}=\mathrm{mean}, \mathrm{SE}=\mathrm{standard}$ error. 
Table C. In vivo toxicity of the $3 \mathrm{R} 4 \mathrm{~F}$ and the $2 \mathrm{R} 4 \mathrm{~F}$ reference cigarettes when smoked under ISO machine smoking conditions.

\begin{tabular}{|c|c|c|c|c|c|c|c|c|}
\hline \multirow{4}{*}{ Organ, Epithelium, Finding } & \multicolumn{4}{|c|}{$\begin{array}{c}\text { 3R4F } \\
\text { ISO }\end{array}$} & \multicolumn{4}{|c|}{$\begin{array}{c}\text { 2R4F } \\
\text { ISO }\end{array}$} \\
\hline & \multicolumn{2}{|c|}{ Male rats } & \multicolumn{2}{|c|}{ Female rats } & \multicolumn{2}{|c|}{ Male rats } & \multicolumn{2}{|c|}{ Female rats } \\
\hline & \multicolumn{2}{|c|}{$200 \mu \mathrm{g} / \mathrm{L}$} & \multicolumn{2}{|c|}{$200 \mu \mathrm{g} / \mathrm{L}$} & \multicolumn{2}{|c|}{$200 \mu \mathrm{g} / \mathrm{L}$} & \multicolumn{2}{|c|}{$200 \mu \mathrm{g} / \mathrm{L}$} \\
\hline & $\mathrm{M}$ & SE & $\mathrm{M}$ & SE & $\mathrm{M}$ & SE & $\mathrm{M}$ & SE \\
\hline \multicolumn{9}{|l|}{ Nose level 1} \\
\hline \multicolumn{9}{|l|}{ Respiratory epithelium } \\
\hline Reserve cell hyperplasia & 4.0 & 0.0 & 4.0 & 0.0 & 4.0 & 0.0 & 4.0 & 0.0 \\
\hline Nasal septum loss of goblet cells & 3.8 & 0.1 & 4.3 & 0.2 & 3.4 & 0.2 & 4.0 & 0.3 \\
\hline Squamous epithelial metaplasia & 4.0 & 0.0 & 4.0 & 0.0 & 4.0 & 0.0 & 4.0 & 0.0 \\
\hline Lamina propria inflammatory cell & & & & & & & & \\
\hline infiltrates & 2.1 & 0.2 & $2.9^{*}$ & 0.4 & 2.2 & 0.4 & 1.8 & 0.2 \\
\hline \multicolumn{9}{|l|}{ Lumen } \\
\hline Exudate & 1.5 & 0.3 & 2.8 & 0.6 & 1.2 & 0.5 & 0.6 & 0.2 \\
\hline \multicolumn{9}{|l|}{ Nose level 2} \\
\hline \multicolumn{9}{|l|}{ Respiratory region } \\
\hline Reserve cell hyperplasia & 2.8 & 0.4 & 3.2 & 0.4 & 3.6 & 0.4 & 2.3 & 0.5 \\
\hline Olfactory region & & & & & & & & \\
\hline Atrophy & 4.6 & 0.3 & 4.2 & 0.4 & 3.6 & 0.5 & 3.8 & 0.5 \\
\hline Squamous metaplasia & 3.2 & 0.5 & 3.0 & 0.6 & 2.6 & 0.6 & 3.0 & 0.5 \\
\hline Loss of nerve bundle & 2.2 & 0.6 & 2.8 & 0.6 & 2.3 & 0.5 & 2.4 & 0.5 \\
\hline Nose level 3 & & & & & & & & \\
\hline Olfactory epithelium & & & & & & & & \\
\hline Atrophy & 3.6 & 0.4 & 3.1 & 0.6 & 2.8 & 0.6 & 3.0 & 0.5 \\
\hline Squamous epithelial metaplasia & 3.6 & 0.4 & 3.1 & 0.6 & 2.6 & 0.6 & 2.8 & 0.6 \\
\hline Lamina propria inflammatory cell & & & & & & & & \\
\hline infiltration & 2.1 & 0.3 & 1.7 & 0.4 & 0.8 & 0.3 & 1.2 & 0.4 \\
\hline Loss of nerve bundle & 3.6 & 0.4 & 3.1 & 0.6 & 2.4 & 0.7 & 2.8 & 0.6 \\
\hline Nose level 4 & & & & & & & & \\
\hline Olfactory epithelium & & & & & & & & \\
\hline Atrophy & 3.6 & 0.4 & 3.1 & 0.6 & 2.8 & 0.6 & 2.9 & 0.6 \\
\hline Squamous epithelial metaplasia & 3.4 & 0.4 & 2.8 & 0.6 & 2.6 & 0.6 & 2.8 & 0.6 \\
\hline Larynx & & & & & & & & \\
\hline Ventral depression & & & & & & & & \\
\hline Squamous epithelial metaplasia & 3.8 & 0.6 & 4.9 & 0.1 & 3.7 & 0.5 & 4.9 & 0.1 \\
\hline Cornification & 5.0 & 0.0 & 2.8 & 0.7 & 5.0 & 0.0 & 5.0 & 0.0 \\
\hline Floor of larynx & & & & & & & & \\
\hline Squamous metaplasia & 5.0 & 0.0 & 5.0 & 0.0 & 5.0 & 0.0 & 5.0 & 0.0 \\
\hline Cornification & 5.0 & 0.0 & 5.0 & 0.0 & 5.0 & 0.0 & 5.0 & 0.0 \\
\hline Vocal cords, lower medial region & & & & & & & & \\
\hline $\begin{array}{l}\text { Squamous epithelial } \\
\text { hyperplasia }\end{array}$ & 1.7 & 0.2 & 1.9 & 0.1 & 2.1 & 0.2 & 1.9 & 0.1 \\
\hline Cornification & 4.0 & 0.6 & 5.0 & 0.0 & 3.9 & 0.5 & 5.0 & 0.0 \\
\hline Vocal cords, upper medial region & & & & & & & & \\
\hline Pseudostratified epithelial & & & & & & & & \\
\hline hyperplasia & 4.0 & 0.4 & 5.0 & 0.0 & 4.5 & 0.2 & 4.9 & 0.1 \\
\hline Cornification & 2.3 & 0.5 & 4.7 & 0.3 & 2.7 & 0.5 & 3.2 & 0.4 \\
\hline Vocal folds & & & & & & & & \\
\hline Squamous epithelium & & & & & & & & \\
\hline Cornification & 4.2 & 0.3 & 4.8 & 0.1 & 3.4 & 0.5 & 5.0 & 0.0 \\
\hline Trachea & & & & & & & & \\
\hline Bifurcation & & & & & & & & \\
\hline Squamous epithelial metaplasia & 1.1 & 0.4 & 1.6 & 0.5 & 2.2 & 0.6 & 1.2 & 0.5 \\
\hline Lung & & & & & & & & \\
\hline Bronchioli & & & & & & & & \\
\hline Goblet cell hyperplasia & 2.6 & 0.7 & 3.6 & 0.5 & 2.4 & 0.7 & 1.7 & 0.5 \\
\hline Lumen & & & & & & & & \\
\hline Alveolar macrophages & 1.7 & 0.2 & 2.2 & 0.2 & 2.1 & 0.1 & 2.1 & 0.2 \\
\hline Pigmented macrophages & 4.5 & 0.4 & 4.7 & 0.3 & 4.0 & 0.5 & 3.8 & 0.5 \\
\hline
\end{tabular}

$M=$ mean scores for histopathological changes after a 90-day inhalation period, $\mathrm{SE}=$ standard error. 
Table D1. In vivo toxicity of the 3R4F reference cigarette when smoked under both the ISO and $\mathrm{HCl}$ machine smoking conditions, male rats.

\begin{tabular}{|c|c|c|c|c|c|c|c|c|c|c|c|c|}
\hline \multirow{3}{*}{ Organ, Epithelium, Finding } & \multicolumn{6}{|c|}{ ISO } & \multicolumn{6}{|c|}{$\mathrm{HCl}$} \\
\hline & \multicolumn{2}{|c|}{$100 \mu \mathrm{g} / \mathrm{L}$} & \multicolumn{2}{|c|}{$150 \mu \mathrm{g} / \mathrm{L}$} & \multicolumn{2}{|c|}{$200 \mu \mathrm{g} / \mathrm{L}$} & \multicolumn{2}{|c|}{$100 \mu \mathrm{g} / \mathrm{L}$} & \multicolumn{2}{|c|}{$150 \mu \mathrm{g} / \mathrm{L}$} & \multicolumn{2}{|c|}{$200 \mu \mathrm{g} / \mathrm{L}$} \\
\hline & $\mathrm{M}$ & SE & $\mathrm{M}$ & SE & $\mathrm{M}$ & SE & $\mathrm{M}$ & SE & $\mathrm{M}$ & SE & M & SE \\
\hline \multicolumn{13}{|l|}{ Nose level 1} \\
\hline Reserve cell hyperplasia & 3.5 & 0.2 & 4.0 & 0.0 & 4.0 & 0.0 & 3.7 & 0.2 & 4.0 & 0.0 & 4.0 & 0.0 \\
\hline Nasal septum loss of goblet cells & 3.2 & 0.3 & 3.9 & 0.2 & 4.4 & 0.2 & 3.1 & 0.3 & 3.9 & 0.1 & 4.1 & 1.2 \\
\hline Squamous epithelial metaplasia & 2.3 & 0.3 & 3.1 & 0.3 & 3.7 & 0.2 & 2.6 & 0.4 & 3.8 & 0.1 & 3.7 & 0.2 \\
\hline \multicolumn{13}{|l|}{$\begin{array}{l}\text { Nose level } 2 \\
\text { Resniratory reaion }\end{array}$} \\
\hline Reserve cell hyperplasia & 1.8 & 0.1 & 2.5 & 0.2 & 3.8 & 0.1 & 1.4 & 0.3 & 3.1 & 0.1 & 3.6 & 0.2 \\
\hline \multicolumn{13}{|l|}{ Olfactory region } \\
\hline Atrophy & 0.4 & 0.2 & 2.4 & 0.6 & 3.6 & 0.5 & 0.1 & 0.1 & 2.4 & 0.6 & 3.6 & 0.6 \\
\hline Squamous metaplasia & 0.0 & 0.0 & 1.3 & 0.5 & 2.0 & 0.5 & 0.0 & 0.0 & 1.2 & 0.5 & 2.3 & 0.5 \\
\hline Loss of nerve bundle & 0.4 & 0.2 & 2.5 & 0.7 & 3.8 & 0.6 & 0.1 & 0.1 & 2.9 & 0.7 & 3.7 & 0.6 \\
\hline \multicolumn{13}{|l|}{$\begin{array}{l}\text { Nose level } 3 \\
\text { Olfactory epithelium }\end{array}$} \\
\hline Atrophy & 0.0 & 0.0 & 1.6 & 0.7 & 2.4 & 0.7 & 0.0 & 0.0 & 1.3 & 0.6 & 2.8 & 0.6 \\
\hline Squamous epithelial metaplasia & 0.0 & 0.0 & 1.3 & 0.6 & 2.0 & 0.6 & 0.0 & 0.0 & 1.0 & 0.5 & 2.4 & 0.5 \\
\hline Loss of nerve bundle & 0.0 & 0.0 & 1.5 & 0.6 & 2.4 & 0.7 & 0.0 & 0.0 & 1.3 & 0.6 & 2.8 & 0.6 \\
\hline \multicolumn{13}{|l|}{$\begin{array}{l}\text { Nose level } 4 \\
\text { Olfactory epithelium }\end{array}$} \\
\hline Atrophy & 0.0 & 0.0 & 1.1 & 0.5 & 2.0 & 0.6 & 0.0 & 0.0 & 1.0 & 0.4 & 2.1 & 0.5 \\
\hline Squamous epithelial metaplasia & 0.0 & 0.0 & 0.9 & 0.4 & 1.9 & 0.6 & 0.0 & 0.0 & 0.4 & 0.3 & 2.0 & 0.5 \\
\hline \multicolumn{13}{|l|}{ Larynx } \\
\hline Squamous epithelial metaplasia & 0.9 & 0.3 & 1.9 & 0.5 & 2.7 & 0.6 & 1.1 & 0.4 & 2.6 & 0.7 & 4.1 & 0.5 \\
\hline Cornification & 0.0 & 0.0 & 0.9 & 0.6 & 1.5 & 0.8 & 0.0 & 0.0 & 1.1 & 0.7 & 2.7 & 0.9 \\
\hline \multicolumn{13}{|l|}{ Floor of larynx } \\
\hline Squamous metaplasia & 4.9 & 0.1 & 5.0 & 0.0 & 5.0 & 0.0 & 5.0 & 0.0 & 5.0 & 0.0 & 5.0 & 0.0 \\
\hline Cornification & 4.7 & 0.3 & 5.0 & 0.0 & 5.0 & 0.0 & 5.0 & 0.0 & 5.0 & 0.0 & 5.0 & 0.0 \\
\hline \multicolumn{13}{|l|}{ Vocal cords, lower medial region } \\
\hline Squamous epithelial hyperplasia & 3.1 & 0.3 & 4.0 & 0.0 & 3.0 & 0.3 & 3.8 & 0.2 & 3.5 & 0.3 & 4.0 & 0.0 \\
\hline Cornification & 1.2 & 0.4 & 3.6 & 0.6 & 3.0 & 0.7 & 2.4 & 0.6 & 2.1 & 0.7 & 4.3 & 0.2 \\
\hline \multicolumn{13}{|l|}{$\begin{array}{l}\text { Vocal cords, upper medial region } \\
\text { Pseudostratified epithelial }\end{array}$} \\
\hline hyperplasia & 1.3 & 0.5 & 4.0 & 0.5 & 3.5 & 0.5 & 2.1 & 0.5 & 2.6 & 0.5 & 4.1 & 0.1 \\
\hline Cornification & 0.0 & 0.0 & 2.7 & 1.0 & 1.5 & 0.9 & 0.0 & 0.0 & 0.6 & 0.6 & 1.0 & 0.6 \\
\hline \multicolumn{13}{|l|}{ Vocal folds } \\
\hline Cornification & 1.22 & 0.5 & 3.0 & 0.5 & 4.1 & 0.3 & 2.4 & 0.7 & 2.8 & 0.8 & 3.9 & 0.3 \\
\hline \multicolumn{13}{|l|}{$\begin{array}{l}\text { Trachea } \\
\text { Bifurcation }\end{array}$} \\
\hline Squamous epithelial metaplasia & 0.0 & 0.0 & 0.1 & 0.1 & 0.2 & 0.1 & 0.0 & 0.0 & 0.3 & 0.3 & 0.6 & 0.3 \\
\hline $\begin{array}{l}\text { Lung } \\
\text { Bronchioli }\end{array}$ & & & & & & & & & & & & \\
\hline Goblet cell hyperplasia & 1.2 & 0.3 & 2.2 & 0.5 & 3.1 & 0.5 & 1.1 & 0.3 & 2.0 & 0.4 & 3.0 & 0.3 \\
\hline Lumen & & & & & & & & & & & & \\
\hline Alveolar macrophages & 1.9 & 0.2 & 1.8 & 0.1 & 2.6 & 0.3 & 1.5 & 0.2 & 2.4 & 0.2 & 2.6 & 0.3 \\
\hline Pigmented macrophage nests & 0.5 & 0.2 & 0.5 & 0.3 & 1.3 & 0.4 & 0.5 & 0.3 & 0.9 & 0.3 & 1.3 & 0.4 \\
\hline
\end{tabular}

$M=$ mean scores for histopathological changes after a 90-day inhalation period, $\mathrm{SE}=$ standard error. 
Table D2. In vivo toxicity of the 3R4F reference cigarette when smoked under both the ISO and $\mathrm{HCl}$ machine smoking conditions, female rats.

\begin{tabular}{|c|c|c|c|c|c|c|c|c|c|c|c|c|}
\hline \multirow{3}{*}{ Organ, Epithelium, Finding } & \multicolumn{6}{|c|}{ ISO } & \multicolumn{6}{|c|}{$\mathrm{HCl}$} \\
\hline & \multicolumn{2}{|c|}{$100 \mu \mathrm{g} / \mathrm{L}$} & \multicolumn{2}{|c|}{$150 \mu \mathrm{g} / \mathrm{L}$} & \multicolumn{2}{|c|}{$200 \mu \mathrm{g} / \mathrm{L}$} & \multicolumn{2}{|c|}{$100 \mu \mathrm{g} / \mathrm{L}$} & \multicolumn{2}{|c|}{$150 \mu \mathrm{g} / \mathrm{L}$} & \multicolumn{2}{|c|}{$200 \mu \mathrm{g} / \mathrm{L}$} \\
\hline & $\mathrm{M}$ & SE & $\mathrm{M}$ & SE & M & SE & M & SE & M & SE & M & SE \\
\hline \multicolumn{13}{|l|}{ Nose level 1} \\
\hline Reserve cell hyperplasia & 3.5 & 0.2 & 4.0 & 0.0 & 4.0 & 0.0 & 3.5 & 0.2 & 4.0 & 0.0 & 4.0 & 0.0 \\
\hline Nasal septum loss of goblet cells & 3.5 & 0.2 & 4.1 & 0.2 & 4.3 & 0.2 & 3.5 & 0.2 & 4.2 & 0.1 & 4.2 & 0.1 \\
\hline Squamous epithelial metaplasia & 2.2 & 0.3 & 3.5 & 0.2 & 3.9 & 0.1 & 2.6 & 0.3 & 3.5 & 0.3 & 4.0 & 0.0 \\
\hline \multicolumn{13}{|l|}{ Nose level 2} \\
\hline Reserve cell hyperplasia & 1.3 & 0.2 & 2.5 & 0.2 & 3.2 & 0.2 & 1.0 & 0.2 & 2.7 & 0.2 & 3.2 & 0.2 \\
\hline \multicolumn{13}{|l|}{ Olfactory region } \\
\hline Atrophy & 0.7 & 0.3 & 3.6 & 0.2 & 4.4 & 0.2 & 1.2 & 0.4 & 3.6 & 0.2 & 4.0 & 0.5 \\
\hline Squamous metaplasia & 0.1 & 0.1 & 2.5 & 0.4 & 2.9 & 0.2 & 0.6 & 0.4 & 2.4 & 0.3 & 2.6 & 0.3 \\
\hline Loss of nerve bundle & 0.8 & 0.4 & 4.1 & 0.1 & 4.7 & 0.2 & 1.6 & 0.6 & 4.2 & 0.1 & 4.0 & 0.5 \\
\hline \multicolumn{13}{|l|}{ Nose level 3} \\
\hline \multicolumn{13}{|l|}{ Olfactory epithelium } \\
\hline Atrophy & 0.0 & 0.0 & 2.4 & 0.5 & 3.6 & 0.4 & 0.3 & 0.2 & 3.0 & 0.4 & 3.4 & 0.4 \\
\hline Squamous epithelial metaplasia & 0.0 & 0.0 & 2.0 & 0.6 & 3.4 & 0.4 & 0.2 & 0.1 & 2.6 & 0.5 & 2.8 & 0.4 \\
\hline Loss of nerve bundle & 0.0 & 0.0 & 2.7 & 0.6 & 3.6 & 0.4 & 0.2 & 0.2 & 3.4 & 0.4 & 3.6 & 0.4 \\
\hline \multicolumn{13}{|l|}{$\begin{array}{l}\text { Nose level } 4 \\
\text { Olfactory epithelium }\end{array}$} \\
\hline Atrophy & 0.0 & 0.0 & 1.4 & 0.4 & 2.7 & 0.3 & 0.0 & 0.0 & 1.8 & 0.4 & 2.5 & 0.5 \\
\hline Squamous epithelial metaplasia & 0.0 & 0.0 & 0.9 & 0.4 & 2.7 & 0.4 & 0.0 & 0.0 & 1.3 & 0.5 & 2.3 & 0.5 \\
\hline \multicolumn{13}{|l|}{$\begin{array}{l}\text { Larynx } \\
\text { Ventral depression }\end{array}$} \\
\hline Squamous epithelial metaplasia & 2.0 & 0.7 & 3.3 & 0.6 & 3.9 & 0.6 & 1.1 & 0.5 & 4.6 & 0.4 & 4.3 & 0.5 \\
\hline Cornification & 1.3 & 0.8 & 2.1 & 0.8 & 3.3 & 0.7 & 0.1 & 0.1 & 4.4 & 0.6 & 3.8 & 0.8 \\
\hline \multicolumn{13}{|l|}{ Floor of larynx } \\
\hline Squamous metaplasia & 5.0 & 0.0 & 5.0 & 0.0 & 5.0 & 0.0 & 5.0 & 0.0 & 5.0 & 0.0 & 5.0 & 0.0 \\
\hline Cornification & 4.7 & 0.2 & 5.0 & 0.0 & 5.0 & 0.0 & 5.0 & 0.0 & 5.0 & 0.0 & 5.0 & 0.0 \\
\hline \multicolumn{13}{|l|}{ Vocal cords, lower medial region } \\
\hline Squamous epithelial hyperplasia & 3.3 & 0.3 & 4.0 & 0.0 & 4.0 & 0.0 & 3.5 & 0.2 & 4.0 & 0.0 & 4.0 & 0.0 \\
\hline Cornification & 2.4 & 0.7 & 4.1 & 0.2 & 4.2 & 0.3 & 2.1 & 0.6 & 4.5 & 0.2 & 4.2 & 0.3 \\
\hline \multicolumn{13}{|l|}{$\begin{array}{l}\text { Vocal cords, upper medial region } \\
\text { Pseudostratified epithelial }\end{array}$} \\
\hline Hyperplasia & 2.1 & 0.6 & 4.0 & 0.2 & 3.8 & 0.4 & 1.0 & 0.3 & 4.3 & 0.4 & 3.8 & 0.6 \\
\hline Cornification & 0.6 & 0.6 & 1.0 & 0.6 & 1.6 & 0.8 & 0.0 & 0.0 & 3.6 & 0.8 & 2.5 & 0.9 \\
\hline \multicolumn{13}{|l|}{ Vocal folds } \\
\hline Cornification & 2.1 & 0.5 & 3.9 & 0.3 & 4.3 & 0.4 & 2.1 & 0.4 & 4.8 & 0.1 & 4.2 & 0.3 \\
\hline $\begin{array}{l}\text { Trachea } \\
\text { Bifurcation }\end{array}$ & & & & & & & & & & & & \\
\hline Squamous epithelial metaplasia & 0.1 & 0.1 & 0.0 & 0.0 & 0.0 & 0.0 & 0.0 & 0.0 & 0.0 & 0.0 & 0.2 & 0.2 \\
\hline $\begin{array}{l}\text { Lung } \\
\text { Bronchioli }\end{array}$ & & & & & & & & & & & & \\
\hline Goblet cell hyperplasia & 0.6 & 0.4 & 2.0 & 0.2 & 2.9 & 0.3 & 1.3 & 0.5 & 2.5 & 0.4 & 2.2 & 0.3 \\
\hline Lumen & & & & & & & & & & & & \\
\hline Alveolar macrophages & 1.5 & 0.2 & 2.1 & 0.2 & 3.0 & 0.2 & 1.6 & 0.2 & 2.3 & 0.2 & 2.8 & 0.2 \\
\hline Pigmented macrophage nests & 0.3 & 0.2 & 1.4 & 0.4 & 2.5 & 0.3 & 0.6 & 0.2 & 1.5 & 0.5 & 1.7 & 0.4 \\
\hline
\end{tabular}

$M=$ mean scores for histopathological changes after a 90-day inhalation period, $\mathrm{SE}=$ standard error. 